\title{
TOI-530b: a giant planet transiting an M-dwarf detected by TESS
}

\author{
Tianjun Gan ${ }^{\circledR},{ }^{1 \star}$ Zitao Lin, ${ }^{2}$ Sharon Xuesong Wang, ${ }^{1 \star}$ Shude Mao, ${ }^{1,3}$ Pascal Fouqué ${ }^{\circledR}, 4,5$ Jiahao Fan, ${ }^{6}$ \\ Megan Bedell, ${ }^{7}$ Keivan G. Stassun ${ }^{\circledR},{ }^{8,9}$ Steven Giacalone, ${ }^{10}$ Akihiko Fukui ${ }^{\circledR},{ }^{11,12}$ Felipe Murgas, ${ }^{12,13}$ \\ David R. Ciardi, ${ }^{14}$ Steve B. Howell, ${ }^{15}$ Karen A. Collins, ${ }^{16}$ Avi Shporer, ${ }^{17}$ Luc Arnold, ${ }^{4}$
} Thomas Barclay, ${ }^{18,19}$ David Charbonneau, ${ }^{16}$ Jessie Christiansen, ${ }^{14}$ Ian J. M. Crossfield, ${ }^{20}$ Courtney D. Dressing, ${ }^{10}$ Ashley Elliott, ${ }^{21}$ Emma Esparza-Borges ${ }^{\circledR},{ }^{12,13}$ Phil Evans, ${ }^{22}$ Crystal L. Gnilka, ${ }^{15}$ Erica J. Gonzales, ${ }^{23}$ Andrew W. Howard, ${ }^{24}$ Keisuke Isogai, ${ }^{25,26}$ Kiyoe Kawauchi ${ }^{\circledR},{ }^{26}$ Seiya Kurita, ${ }^{27}$ Beibei Liu ${ }^{\circledR},{ }^{28}$ John H. Livingston ${ }^{\circledR},{ }^{29}$ Rachel A. Matson ${ }^{\circledR},{ }^{30}$ Norio Narita, ${ }^{11,12,31,32}$ Enric Palle, ${ }^{12,13}$ Hannu Parviainen, ${ }^{12,13}$ Benjamin V. Rackham ${ }^{\circledR},{ }^{17,33} \dagger$ David R. Rodriguez, ${ }^{34}$ Mark Rose, ${ }^{15}$ Alexander Rudat, ${ }^{17}$ Joshua E. Schlieder, ${ }^{18}$ Nicholas J. Scott, ${ }^{15}$ Michael Vezie, ${ }^{17}$ George R. Ricker, ${ }^{17}$ Roland Vanderspek, ${ }^{17}$ David W. Latham, ${ }^{16}$ Sara Seager, ${ }^{17,33,35}$ Joshua N. Winn ${ }^{36}$ and Jon M. Jenkins ${ }^{15}$

Affiliations are listed at the end of the paper

Accepted 2021 October 7. Received 2021 September 26; in original form 2021 July 21

\begin{abstract}
We report the discovery of TOI-530b, a transiting Saturn-like planet around an M0.5V dwarf, delivered by the Transiting Exoplanet Survey Satellite (TESS). The host star is located at a distance of $147.7 \pm 0.6 \mathrm{pc}$ with a radius of $R_{*}=0.54 \pm 0.03 R_{\odot}$ and a mass of $M_{*}=0.53 \pm 0.02 \mathrm{M}_{\odot}$. We verify the planetary nature of the transit signals by combining ground-based multiwavelength photometry, high-resolution spectroscopy from SPIRou as well as high-angular-resolution imaging. With $V=$ $15.4 \mathrm{mag}$, TOI-530b is orbiting one of the faintest stars accessible by ground-based spectroscopy. Our model reveals that TOI$530 \mathrm{~b}$ has a radius of $0.83 \pm 0.05 R_{J}$ and a mass of $0.37 \pm 0.08 M_{J}$ on a $6.39-\mathrm{d}$ orbit. TOI-530b is the sixth transiting giant planet hosted by an M-type star, which is predicted to be infrequent according to core accretion theory, making it a valuable object to further study the formation and migration history of similar planets. Furthermore, we identify a potential dearth of hot massive giant planets around M-dwarfs with separation distance smaller than 0.1 au and planet-to-star mass ratio between $2 \times 10^{-3}$ and $10^{-2}$. We also find a possible correlation between hot giant planet formation and the metallicity of its parent M-dwarf. We discuss the potential formation channel of such systems.
\end{abstract}

Key words: planets and satellites: detection-planets and satellites: gaseous planets - planets and satellites: individual: TIC 387690507, TOI 530 - stars: low-mass.

\section{INTRODUCTION}

M-dwarfs are popular targets for exoplanet research. First, radial velocity (RV) variations induced by the planets around M-dwarfs are more significant than those around solar-like stars, making it possible to obtain precise mass measurement towards the terrestrial planet end of the mass distribution. Second, their small stellar radii lead to a large planet-to-star radius ratio, which favours transit detections and further photometric follow-up observations. Planets around Mdwarfs are also attractive sources for atmospheric characterization through transmission or emission spectroscopy (Batalha et al. 2018; Kempton et al. 2018) as they yield a higher signal-to-noise ratio than equivalent systems with other types of hosts (e.g. LHS 3844b, Kreidberg et al. 2019; Vanderspek et al. 2019; Diamond-Lowe et al.

\footnotetext{
^E-mail: gtj18@mails.tsinghua.edu.cn (TG); sharonw@mail.tsinghua.edu.cn (SXW) $\dagger 51$ Pegasi b Fellow.
}

2020). Finally, due to the low stellar luminosity (typically $L<$ $\left.0.1 L_{\odot}\right)$, the habitable zone of M-dwarfs is closer to the host star when compared with luminous stars (e.g. TOI-700d, Gilbert et al. 2020; Rodriguez et al. 2020), which offers particular advantages to look for planets with potential biosignatures.

Over the last two decades, more than a thousand transiting giant planets (defined as $M_{\mathrm{p}} \geq 0.3 M_{\mathrm{J}}$ ) have been discovered thanks to successful ground-based surveys, including HATNet (Bakos et al. 2004), SuperWASP (Pollacco et al. 2006), KELT (Pepper et al. 2007, 2012) and NGTS (Chazelas et al. 2012; Wheatley et al. 2018) as well as space transit missions like CoRoT (Baglin et al. 2006), Kepler (Borucki et al. 2010), and $K 2$ (Howell et al. 2014). However, even though M-dwarfs are the most abundant stellar population in our Milky Way (Henry et al. 2006), only five giant planets have been confirmed to transit them: Kepler-45b (Johnson et al. 2012), HATS$6 \mathrm{~b}$ (Hartman et al. 2015), NGTS-1b (Bayliss et al. 2018), HATS71b (Bakos et al. 2020), and TOI-1899b (Cañas et al. 2020). The deficiency of such systems is thought to be caused by the failed growth of a massive core to start runaway accretion before the 

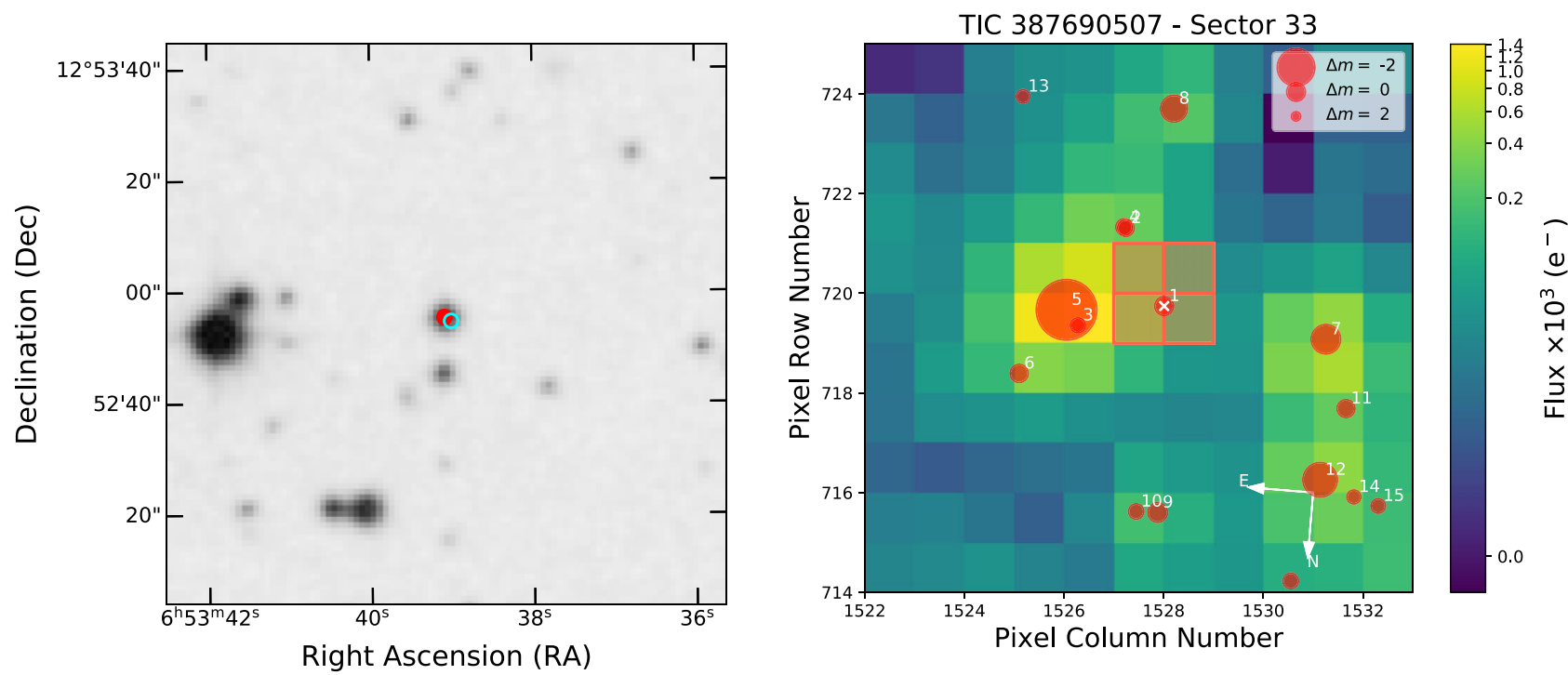

Figure 1. Left-hand panel: The POSS2 blue image of TOI-530 taken in 1996. The centre red dot is the target star in this image and the cyan circle shows its current position, which rules out the unassociated distant eclipsing binary scenario. Right-hand panel: Target pixel file (TPF) of TOI-530 in TESS Sector 33 (created with tpfplotter, Aller et al. 2020). Different sizes of red circles represent different magnitudes in contrast with TOI-530 ( $\Delta m$ ). The aperture used to extract the photometry is overplotted with a red-square region.

gaseous protoplanetary disc dissipates due to the low surface density (Laughlin, Bodenheimer \& Adams 2004; Ida \& Lin 2005; Kennedy \& Kenyon 2008; Liu \& Ji 2020). Indeed, previous statistical studies of the occurrence rates from Kepler show that planets with radii between 1 and $4 R_{\oplus}$ are frequent around low-mass stars (Dressing \& Charbonneau 2013, 2015; Hardegree-Ullman et al. 2019). Some of these small planets are possibly the bare cores of failed gas giants. Nevertheless, microlensing surveys have found plenty of cold Jupiters $(a \gtrsim 1 \mathrm{au})$ around M-dwarfs (e.g. Zang et al. 2018), which hints that outer giant planets are probably not rare. A handful of such cases have also been reported by long-term RV observations (e.g. GJ 876b, Marcy et al. 2001; GJ 849b, Butler et al. 2006; GJ 179b, Howard et al. 2010; HIP 79431b, Apps et al. 2010). Gravitational instability is speculated to be the alternative formation mechanism responsible for the surprising number of long-period gas giants around M-dwarfs (Boss 2000; Morales et al. 2019). But it is still unclear how these short-period $(P \lesssim 30 \mathrm{~d})$ transiting gas giants were formed, and whether they have migrated into their current orbits due to the lack of such systems. Therefore, establishing a well-characterized sample of this kind of planet is an important step to study their formation. Further Rossiter-McLaughlin (McLaughlin 1924; Rossiter 1924) or Doppler tomography (Marsh 2001) measurements could reveal the obliquity of these systems, providing important clues about the dynamical history of the planets (e.g. Albrecht et al. 2012).

The Transiting Exoplanet Survey Satellite (TESS, Ricker et al. 2014, 2015), which has performed a two-year all-sky survey, offers exciting opportunities to increase the number of transiting giant planets around M-dwarfs. Although TESS has already identified several such planet candidates, the intrinsic faintness of their hosts $(V \gtrsim 15$ mag) challenges most ground-based optical spectroscopic facilities to further conduct detailed RV follow-up observations. Some efforts have already been made to validate those planets through multicolour transit modelling and phase curve analysis (e.g. TOI-519b, Parviainen et al. 2021). The new-generation near-infrared spectrograph SPIRou on the Canada-France-Hawaii-Telescope (CFHT) opens a new window to characterize planets around faint stars (Artigau et al. 2014a; Donati et al. 2020). It was designed to perform high-precision velocimetry and spectropolarimetry studies. Early observations from
SPIRou have shown that it can reach $2 \sim 10 \mathrm{~m} \mathrm{~s}^{-1}$ precision for stars with $H<10$ mag (Moutou et al. 2020; Artigau et al. 2021; Klein et al. 2021). Although simulations predict that SPIRou could reach $<2 \mathrm{~m} \mathrm{~s}^{-1} \mathrm{RV}$ precision for inactive M-dwarfs with $J<10$ mag (see fig. 5 in Cloutier et al. 2018), precision for faint stars has yet to be determined observationally.

Here, we report the discovery of a new transiting giant planet around an M-dwarf star TOI-530. We present RV measurements from SPIRou that allow us to obtain a precise companion mass and thus confirm its planetary nature. The rest of the paper is organized as follows. We describe all space and ground-based observational data used in this work in Section 2. Section 3 presents the stellar properties. In Section 4, we show our analysis of the light curves and RV data. We discuss the prospects of future atmospheric characterization of TOI-530b and its potential formation channel in Section 5. We conclude with our findings in Section 6.

\section{OBSERVATIONS}

\subsection{TESS photometry}

TOI-530 was observed by TESS on its Camera 1 with the two-minute cadence mode in Sector 6 during the primary mission and Sector 33 during the extended mission. The current data span from 2018 December 15 to 2021 January 13, consisting of 14830 and 17547 measurements, respectively. The target will be revisited in Sectors 44-45 between 2021 October 12 and December 2. Fig. 1 shows the POSS2 and TESS images centred on TOI-530.

The photometric data from Sector 6 were initially reduced by the Science Processing Operations Center (SPOC; Jenkins et al. 2016) pipeline, developed based on the Kepler mission's science pipeline. The simple aperture photometry (SAP) flux time series was corrected for instrumental and systematic effects, and for crowding and dilution with the Presearch Data Conditioning (PDC; Stumpe et al. 2012, 2014; Smith et al. 2012) module. Transit signals were searched using the Transiting Planet Search (TPS; Jenkins 2002; Jenkins et al. 2017) algorithm on 17 February 2019, yielding a strong transit signal at a period of $\sim 6.39 \mathrm{~d}$ and a transit duration of $\sim 2.5 \mathrm{~h}$. The transit 
Sector 6
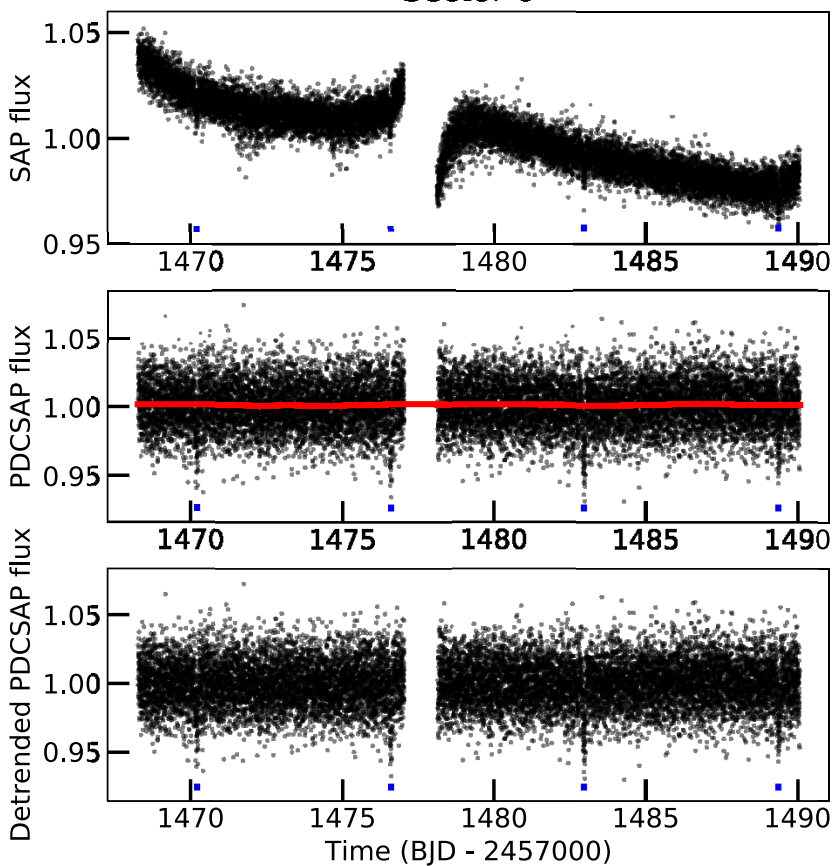

Sector 33
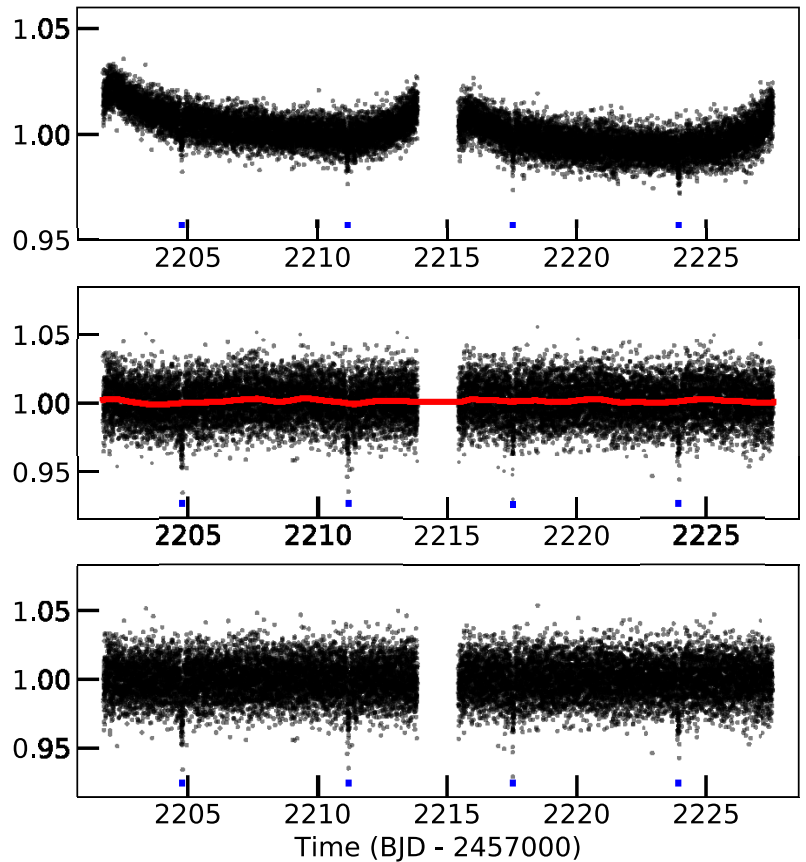

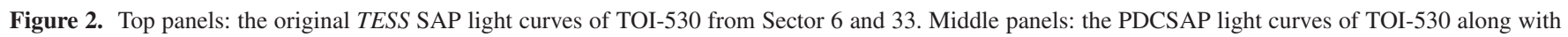
the best-fitting GP model shown as red solid lines. Bottom panels: the detrended PDCSAP light curves. The transits of TOI-530b are marked in blue ticks.

Table 1. Ground-based photometric follow-up observations for TOI-530.

\begin{tabular}{|c|c|c|c|c|c|c|c|c|}
\hline Telescope & Camera & Filter & Pixel scale & Aperture size (pixel) & Coverage & Date & Duration (min) & Total exposures \\
\hline El Sauce $(0.36 \mathrm{~m})$ & STT 1603 & $R_{\mathrm{c}}$ & 1.47 & 4 & Egress & 2019 November 21 & 183 & 59 \\
\hline $\operatorname{TCS}(1.52 \mathrm{~m})$ & MuSCAT2 & $g$ & 0.44 & 9.8 & Egress & 2020 January 4 & 237 & 305 \\
\hline $\operatorname{TCS}(1.52 \mathrm{~m})$ & MuSCAT2 & $r$ & 0.44 & 9.8 & Egress & 2020 January 4 & 237 & 456 \\
\hline $\operatorname{TCS}(1.52 \mathrm{~m})$ & MuSCAT2 & $i$ & 0.44 & 9.8 & Egress & 2020 January 4 & 237 & 456 \\
\hline $\operatorname{TCS}(1.52 \mathrm{~m})$ & MuSCAT2 & $z_{\mathrm{s}}$ & 0.44 & 9.8 & Egress & 2020 January 4 & 237 & 238 \\
\hline NAOJ (1.88 m) & MuSCAT & $g$ & 0.36 & 18 & Egress & 2020 March 2 & 177 & 321 \\
\hline NAOJ $(1.88 \mathrm{~m})$ & MuSCAT & $r$ & 0.36 & 18 & Egress & 2020 March 2 & 177 & 268 \\
\hline NAOJ $(1.88 \mathrm{~m})$ & MuSCAT & $z_{\mathrm{s}}$ & 0.36 & 18 & Egress & 2020 March 2 & 177 & 474 \\
\hline
\end{tabular}

signature and pixel data passed all the validation tests (Twicken et al. 2018; Li et al. 2019; Guerrero et al. 2021), including locating the source of the transit signature to within 1-3 arcsec of the target star, and no further transiting planet signatures were identified in a search of the residual light curve. The vetting results were reviewed by the TESS Science Office (TSO) and issued an alert for TOI-530b as a planet candidate on 28 March 2019.

We downloaded the Presearch Data Conditioning Simple Aperture Photometry (PDCSAP) light curve from the Mikulski Archive for Space Telescopes (MAST ${ }^{1}$ ) using the lightkurve package (Lightkurve Collaboration et al. 2018; Barentsen et al. 2019). Combining the data sets of two sectors, we conducted an independent transit search by utilizing the Transit Least Squares (TLS; Hippke \& Heller 2019) algorithm, which is an advanced version of Box Least Square (BLS; Kovács, Zucker \& Mazeh 2002), after smoothing the full light curve with a median filter. We recovered the $6.387 \mathrm{~d}$ transits with a signal detection efficiency (SDE) of $\sim 50$. After subtracting the TLS model from the TESS data, we did not find any other significant transit signals existing in the light curve. We detrended the raw TESS light curve by fitting a Gaussian Process (GP) model with a Matérn$3 / 2$ kernel using the celerite package (Foreman-Mackey et al. 2017), after masking out all in-transit data. We show the reprocessed light curve in Fig. 2.

\subsection{Ground-based photometry}

We collected a series of ground-based observations of TOI-530, as part of the TESS Follow-up Observing Program (TFOP ${ }^{2}$ ), to (1) confirm the transit signal on target and rule out nearby eclipsing binary (EB) scenario; (2) examine the chromaticity; and (3) refine the transit ephemeris and radius measurement. These observations were scheduled with the help of the TESS Transit Finder (TTF), which is a customized version of the Tapir software package (Jensen 2013). Due to the observational constraints, unfortunately, we only covered the egress of the event. We summarize the details in Table 1 and describe individual observations below. We show the raw and detrended ground-based light curves in Fig. 3 (see Section 4.1.2). 

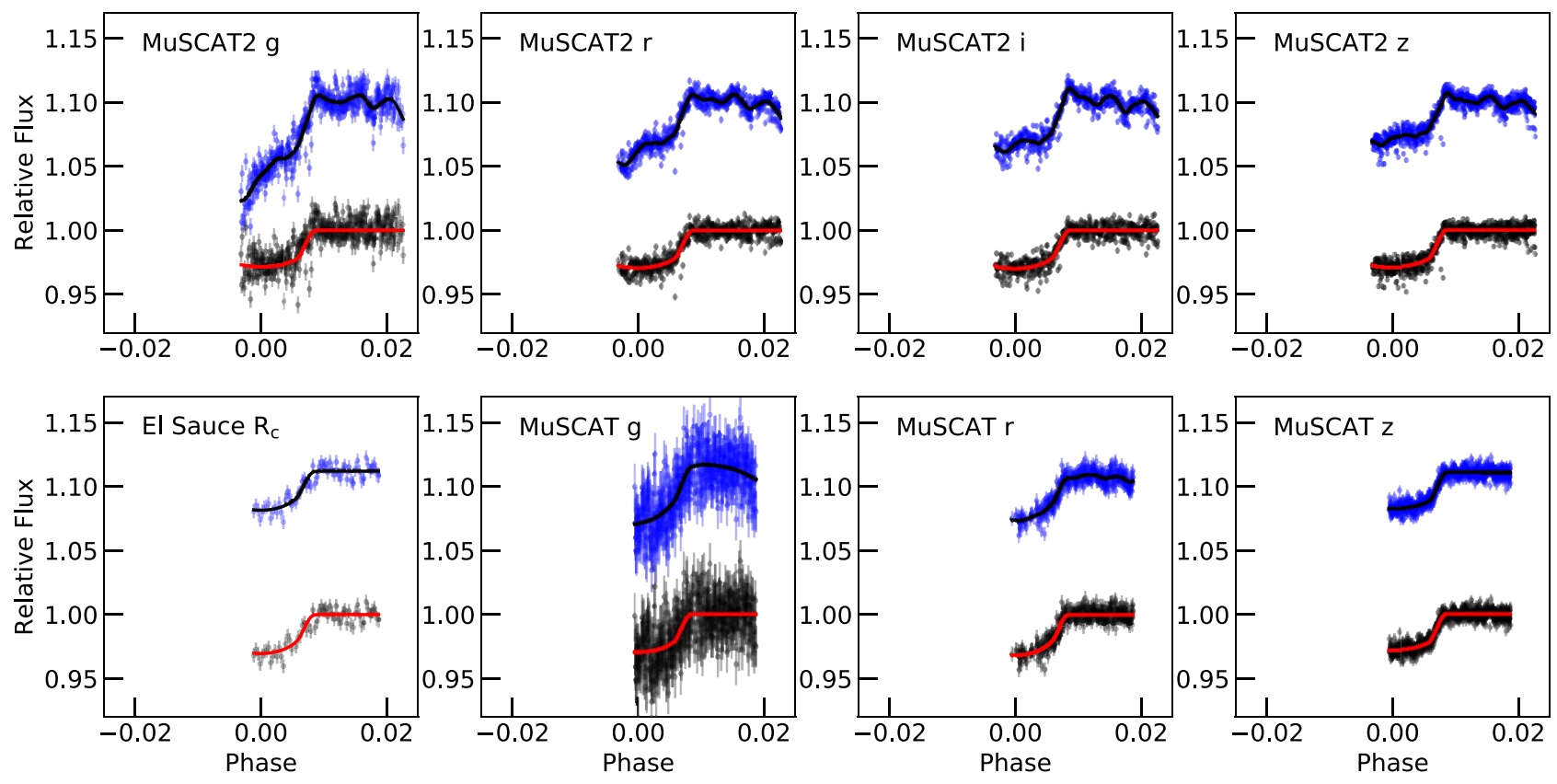

Figure 3. Ground-based light curves for all available instrument. The blue dots are the raw data while the black solid line represents the best fit GP + transit model. The black dots are results after subtracting the GP model (i.e. detrended data). We use these detrended light curves in the final joint-fit (see Section 4.3).

\subsubsection{El Sauce}

An egress was observed on UT 2019 November 21 in the $R_{\mathrm{c}}$ band using the Evans telescope $(0.36 \mathrm{~m})$ at the El Sauce Observatory, Chile. The STT 1603 camera has a pixel scale of 1.47 arcsec per pixel. We acquired a total of 65 images over $205 \mathrm{~min}$. Photometric analysis was carried out using AstroImageJ (Collins et al. 2017) with an uncontaminated aperture of 5.88 arcsec. We excluded all nearby stars within 1 arcmin as the source causing the TESS signal with brightness difference down to $\Delta T \sim 4.1 \mathrm{mag}$, and confirmed the signal on target.

\subsubsection{MuSCAT2}

We observed an egress of TOI-530b on the night of UT 2020 January 4 with the multicolour imager MuSCAT2 (Narita et al. 2019) mounted on the $1.52 \mathrm{~m}$ Telescopio Carlos Sánchez at Teide Observatory, Tenerife, Spain. MuSCAT2 has a field of view of $7.4 \times 7.4 \operatorname{arcmin}^{2}$ with a pixel scale of $0.44 \operatorname{arcsec}_{\text {pixel }}{ }^{-1}$ and is able to obtain simultaneous photometry in four bands $(g, r, i$, and $z_{\mathrm{s}}$ ). The observations were made with the telescope in optimal focus and the exposure times for each band were $45 \mathrm{~s}$ for $g, 30 \mathrm{~s}$ for $r$ and $i$, and $20 \mathrm{~s}$ for $z_{\mathrm{s}}$ band. The data were calibrated using standard procedures (dark and flat calibration). Aperture photometry and transit light curve fit were performed using MuSCAT2 pipeline (Parviainen et al. 2020); the pipeline finds the aperture that minimizes the photometric dispersion while fitting a transit model including instrumental systematic effects present in the time series.

\subsubsection{MUSCAT}

We observed an egress of TOI-530b on UT 2020 March 2 in $g, r$, and $z_{\mathrm{s}}$ bands, using the multiband imager MuSCAT (Narita et al. 2015) mounted on the 188-cm telescope of National Astronomical Observatory of Japan at the Okayama Astro-Complex, Japan. MuSCAT has three CCD cameras, each having a pixel scale of 0.361 arcsec pixel $^{-1}$ and a field of view of $6.1^{\prime} \times 6.1$. We acquired 321, 268, and 474 images with exposure times of 30, 30, and $20 \mathrm{~s}$ in $g, r$, and $z_{\mathrm{s}}$ bands, respectively. The data were dark-subtracted and flatfield corrected in a standard manner. Aperture photometry was then performed on the reduced images using a custom pipeline (Fukui et al. 2011). The radius of the photometric aperture was chosen to be 18 pixels $(6.5 \mathrm{arcsec})$ for all bands so that the photometric dispersion was minimized.

\subsection{Spectroscopic observations}

\subsubsection{IRTF}

We observed TOI-530 on UT 2019 April 23 with the uSpeX spectrograph (Rayner et al. 2003, 2004) on the 3-m NASA Infrared Telescope Facility (IRTF). Our data were collected in the SXD mode using the $0.3 \times 15 \operatorname{arcsec}^{2}$ slit and covers a wavelength range of $0.7-2.55 \mu \mathrm{m}$. The data were reduced using the Spextool pipeline (Cushing, Vacca \& Rayner 2004). After reducing, we RV-correct our spectrum using tellrv (Newton et al. 2014), with which we estimate a systemic RV of $-26 \pm 5 \mathrm{~km} \mathrm{~s}^{-1}$. By comparing our spectrum to those provided by the IRTF library (Rayner, Cushing \& Vacca 2009), we determine that our spectrum best matches that of a star of spectral type M0.5V (See Fig. 4). Lastly, we calculate the metallicity of TOI-530 following the relations defined in Mann et al. (2013) for cool dwarfs with spectral types between K7 and M5. In performing this calculation, we opted to only use the $K s$-band spectrum, as Dressing et al. (2019) found $K s$-band spectra to produce more reliable metallicities and suffer less telluric contamination than $H$-band spectra. Our analysis yield metallicities of $[\mathrm{Fe} / \mathrm{H}]=$ $0.376 \pm 0.095$ and $[\mathrm{M} / \mathrm{H}]=0.218 \pm 0.092$. 


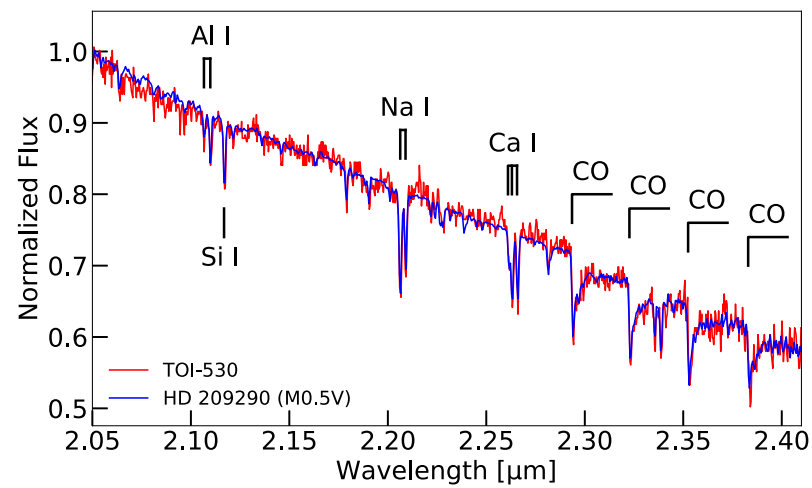

Figure 4. Renormalized SpeX spectrum of TOI-530 (red line) and the comparison spectrum (blue line) taken from the IRTF library (Rayner et al. 2009). The strong atomic features are marked based on the results from Cushing, Rayner \& Vacca (2005). The NIR spectrum of TOI-530 corresponds to a spectral type of $\mathrm{M} 0.5 \mathrm{~V}$.

\subsubsection{CFHT/SPIRou}

We monitored TOI-530 over five epochs between UT 2020 September 26 and October 5 using SPIRou (standing for SpectroPolarimètre InfraROUge), which is a new-generation high-resolution (64 000) fiber-fed spectrograph with polarimetric and precision velocimetry capacities, installed at CFHT in 2018 (Artigau et al. 2014a; Donati et al. 2018). It has a large bandwidth (from 0.95 to $2.5 \mu \mathrm{m}$ ) allowing the detection of several stellar lines in a single shot thus enhancing the precision of the measurement of the stellar RV. For each night, we obtained three sequences, with 975 s exposure time for each. The spectroscopic data were reduced using the standard data reduction pipeline (APERO; Cook et al., in preparation), which performs the data calibration and corrects the telluric and night-sky emission (Artigau et al. 2021). For the Night-sky emission, it is corrected using a principal component analysis (PCA) model of $\mathrm{OH}$ emission constructed from a library of high-SNR sky observations (Artigau et al. 2014b). The telluric absorption is corrected using a PCAbased approach on residuals after fitting for a basic atmospheric transmission model (TAPAS, Bertaux et al. 2014).

We extracted the RVs from the telluric-substracted SPIRou data using two pipelines: wobble by Bedell et al. (2019) and for validation purposes, our own template-based cross-correlation code following the basic algorithms outlined by Zechmeister et al. (2018). Our RV extraction for SPIRou, in general, is detailed in another paper (Lin et al., in preparation), and here we briefly describe our work on TOI-530 and the associated validation tests.

We extracted RVs using the portion of the spectra in the $Y, J, H$, and $K$ bands, namely, orders 1-11, 17-22, 29-37, and 43-44 in SPIRou data. The eliminated orders were visually examined to have heavy telluric absorption and poor telluric removal. We removed the residual emission lines by performing $3 \sigma$ clipping from the local median flux and normalized the spectra using the Alpha-shape Fitting to Spectrum algorithm (AFS) by Xu et al. (2019). Additional emission lines were masked out by selecting pixels that are clearly above the local continuum by more than 20 per cent. We had visually examined our emission-line-subtracted and normalized spectra to tune the normalization and line removal parameters to ensure that there was no systematics or oversubtraction of emission lines in this process.

Then we measured the RVs from the processed spectra using wobble, and the final RVs are listed in Table 2. Briefly, wobble constructs a linear model to infer the stellar and time-varying telluric
Table 2. SPIRou RV measurements of TOI-530. Each observation took an exposure time of $975 \mathrm{~s}$.

\begin{tabular}{lcc}
\hline BJD $_{\text {TDB }}$ & $\mathrm{RV}\left(\mathrm{m} \mathrm{s}^{-1}\right)$ & $\sigma_{\mathrm{RV}}\left(\mathrm{m} \mathrm{s}^{-1}\right)$ \\
\hline 2459119.08206 & -61.40 & 15.26 \\
2459119.09361 & -108.96 & 11.90 \\
2459119.10515 & -121.31 & 9.39 \\
2459120.06581 & -26.38 & 11.09 \\
2459120.07736 & 15.54 & 15.63 \\
2459120.08897 & -20.90 & 12.12 \\
2459123.06563 & -65.09 & 15.70 \\
2459123.07718 & -63.84 & 15.60 \\
2459123.08873 & -102.48 & 13.46 \\
2459127.07895 & 111.67 & 20.29 \\
2459127.09089 & 101.52 & 14.63 \\
2459127.10289 & 124.66 & 14.08 \\
2459128.06393 & 131.33 & 14.84 \\
2459128.07548 & 17.58 & 15.10 \\
2459128.08703 & 75.06 & 18.71 \\
\hline
\end{tabular}

spectra without requiring any prior knowledge on them, while solving for the RV at each epoch. We have adjusted the parameters within wobble, including the convergence step sizes and steps and the template grid size, following the directions in the published codes by Bedell et al. (2019). We had adjusted the regularization parameters which regularize the template-fitting process, but due to the low SNR of the spectra, the tuned parameters did not converge well and wobble's default regularization parameters performed better. The RV results on planet TOI-530b using wobble are marginally consistent with each other within $1 \sigma$ regardless of whether we apply additional masking to the spectral regions with telluric contamination or not, so we adopt the results with this additional telluric masking in case the residual tellurics do induce additional systematics in the data. We used a telluric mask adopted by the CARMENES NIR arm as published by Zechmeister et al. (2018).

To verify our RVs reported by wobble, we also extracted RVs from three months (June, October, and December of 2020) of publicly available data on SPIRou's RV standard star, GL 846. A quick extraction over the $H$ band, where the SNRs are the highest and the telluric removal appears to be the best, we obtained an RV scatter of $11 \mathrm{~m} \mathrm{~s}^{-1}$, consistent with the results from the SPIRou team.

In addition to the planet's signal, we also see a linear trend in the RVs of TOI-530 (see Section 4.2 for more details). It is unclear for now whether this RV trend is of astrophysical or systematic origin. On one hand, we do not see such trends in the RVs of GL 846, indicating that the long-term stability of SPIRou is better than $\sim 10 \mathrm{~m} \mathrm{~s}^{-1}$ at least. On the other hand, we see a linear trend of roughly $160 \mathrm{~m} \mathrm{~s}^{-1}$ over two weeks in the RVs of GL 436 extracted by wobble using the same set of SPIRou data as in Donati et al. (2020), although we have recovered the planet's RV signal accurately to the same level of precision (nightly binned residual RMS $<3 \mathrm{~m} \mathrm{~s}^{-1}$ ) as Donati et al. (2020). ${ }^{3}$ This linear trend, however, was not reported by Donati et al. (2020) or the original discovery paper of GL $436 \mathrm{~b}$ by Butler et al. (2004), which had RVs over the course of four years. Even if

\footnotetext{
${ }^{3}$ We note that we have also extracted RVs using the SERVAL pipeline by Zechmeister et al. (2018), which reports an RV scatter of $\sim 9 \mathrm{~m} \mathrm{~s}^{-1}$ on GL 846 and the same results on GL 436 with the same linear trend as using wobble. SERVAL, however, could not process the TOI-530 spectra due to their low SNRs, so we do not report our SERVAL results in this work. We will publish our SPIRou pipelines and the relevant results in an upcoming paper (Lin et al., in preparation).
} 

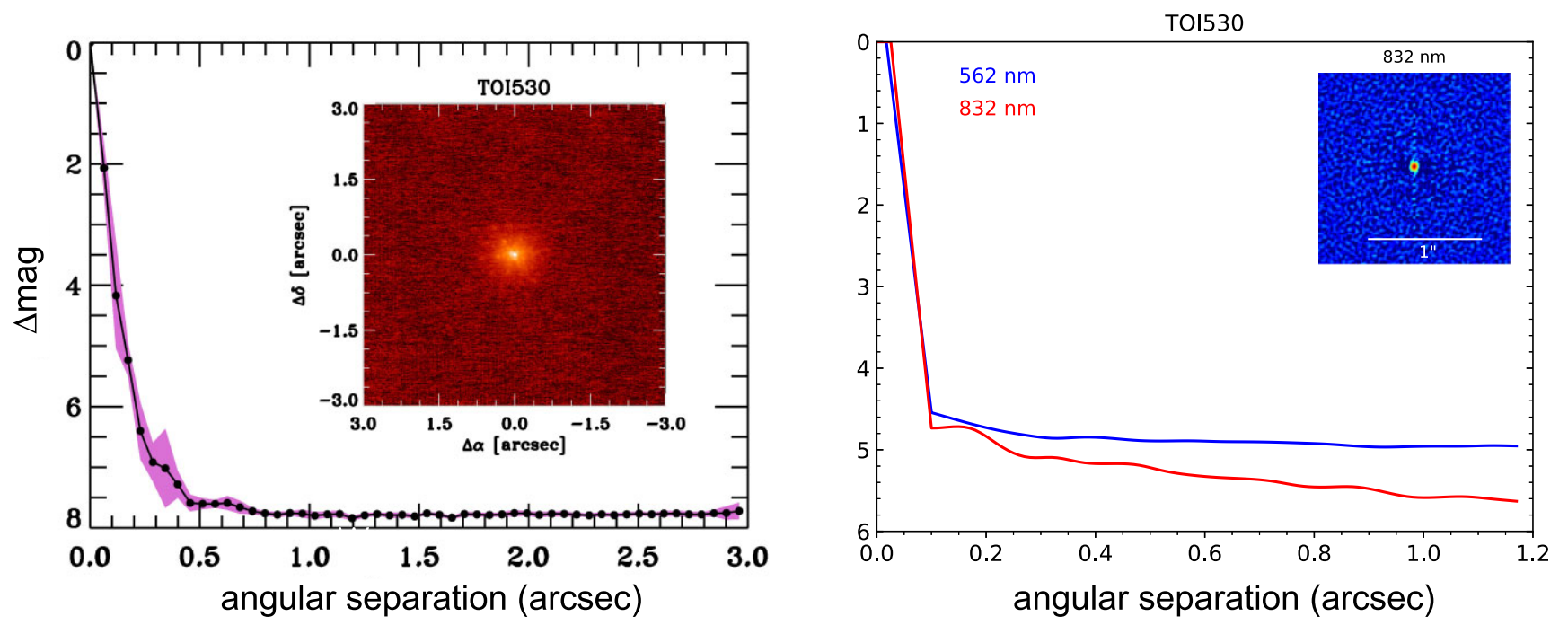

Figure 5. Left-hand panel: NIRC2 AO image (inset) and $K_{s}$-band contrast curve for TOI-530. The black line is the $5 \sigma$ sensitivity limit. The shaded purple region represents the azimuthal dispersion $(1 \sigma)$ of the contrast determinations. Right-hand panel: The $5 \sigma$ 'Alopeke speckle imaging contrast curves in both filters as a function of the angular separation out to $1.2 \mathrm{arcsec}$, the end of speckle coherence. The inset shows the reconstructed 832-nm image with a 1-arcsec scale bar. The star, TOI-530, was found to have no close companions to within the contrast levels achieved.

the signal is from uncorrected instrumental or pipeline systematics, our results on GL 436b suggest that this is unlikely to affect our measurement on the mass of TOI-530b. More RV data would help address the origin of this linear trend, and we will conduct more tests on our SPIRou pipelines in future work (Lin et al. in preparation).

\subsection{High angular resolution imaging}

If an exoplanet host star has a spatially close companion, that companion (bound or line of sight) can create a false-positive transit signal if it is, for example, an EB. For small stars and large planets, this is an especially important check to make, due to the paucity of giant planets orbiting M stars. 'Third-light' flux from the close companion star can lead to an underestimated planetary radius if not accounted for in the transit model (Ciardi et al. 2015) and cause non-detections of small planets residing with the same exoplanetary system (Lester et al. 2021). Additionally, the discovery of close, bound companion stars, which exist in nearly one-half of FGK type stars (Matson et al. 2018) and less so for M class stars, provides crucial information toward our understanding of exoplanetary formation, dynamics and evolution (Howell et al. 2021). Thus, to search for close-in bound companions unresolved in TESS or other ground-based follow-up observations, we obtained high-resolution imaging observations of TOI-530.

\subsubsection{Keck/NIRC2 adaptive optics imaging}

We observed TOI-530 with infrared high-resolution adaptive optics (AO) imaging at Keck Observatory (Ciardi et al. 2015; Schlieder et al. 2021) on UT 2019 April 7. The observations were made with the NIRC2 instrument on Keck-II behind the natural guide star AO system. The standard three-point dither pattern was used to avoid the lower left-hand quadrant of the detector which is typically noisier than the other three quadrants. The dither pattern step size was 3 arcsec and it was repeated twice, with each dither offset from the previous one by 0.5 arcsec.
The observations were taken in the broad-band $\mathrm{K}\left(\lambda_{o}=2.1956\right.$ $\mu \mathrm{m} ; \Delta \lambda=0.336 \mu \mathrm{m}$ ) with an integration time of $4 \mathrm{~s}$ per frame for a total on-source integration time of $36 \mathrm{~s}$. The camera was in the narrow-angle mode with a full field of view of $10 \mathrm{arcsec}$ and a pixel scale of approximately 0.009942 arcsec per pixel. The Keck AO observations show no additional stellar companions were detected to within a resolution $\sim 0.056$-arcsec FWHM. The sensitivities of the final combined $\mathrm{AO}$ image were determined by injecting simulated sources azimuthally around the primary target every $20^{\circ}$ at radial separations of integer multiples of the FWHM of the central source (Furlan et al. 2017). The brightness of each injected source was scaled until standard aperture photometry detected it with $5 \sigma$ significance. The resulting brightness of the injected sources relative to the target TOI-530 was regarded as the contrast limits at that injection location. The final $5 \sigma$ limit at each separation was determined from the average of all of the determined limits at that separation while the uncertainty was given by the RMS dispersion of the results for different azimuthal slices at a given radial distance. We show the $2-\mu \mathrm{m}$ sensitivity curve on the left-hand panel of Fig. 5 along with an inset image zoomed to primary target, which shows no other companion stars.

\subsubsection{Gemini-North speckle imaging}

TOI-530 was observed on 2020 February 17 UT using the 'Alopeke speckle instrument on the Gemini North 8-m telescope. 4 'Alopeke provides simultaneous speckle imaging in two bands (562 and $832 \mathrm{~nm}$ ) with output data products including a reconstructed image with robust contrast limits on companion detections (e.g. Howell et al. 2016). Ten sets of $1000 \times 0.06$-s exposures were collected and subjected to Fourier analysis in our standard reduction pipeline (Howell et al. 2011). The right-hand panel of Fig. 5 shows our final contrast curves and the $832 \mathrm{~nm}$ reconstructed speckle image. We find that TOI-530 is a single star with no companion brighter than 5-6 mag below that of the target star (earlier than $\sim \mathrm{M} 4.5 \mathrm{~V}$ ) from the

\footnotetext{
${ }^{4} \mathrm{https}: / / \mathrm{www} . g e m i n i . e d u / s c i o p s /$ instruments/alopeke-zorro/
} 
diffraction limit (20 mas) out to 1.2 arcsec. At the distance of TOI$530(d=148 \mathrm{pc})$, these angular limits correspond to spatial limits of 3-178 au.

\section{STELLAR CHARACTERIZATION}

We first use 2MASS observed $m_{K}$ and the parallax from Gaia EDR3 to calculate the absolute magnitude, of which we obtain $M_{K}=$ $5.39 \pm 0.02 \mathrm{mag}$. We then estimate the stellar radius following the polynomial relation between $R_{*}$ and $M_{K}$ derived by Mann et al. (2015), and we find $R_{*}=0.54 \pm 0.02 R_{\odot}$, assuming a typical uncertainty of 3 percent (see table 1 in Mann et al. 2015). For comparison, we also estimate the stellar radius $R_{*}=0.55 \pm 0.03 R_{\odot}$ based on the angular diameter relation in Boyajian, van Belle \& von Braun (2014), consistent with our previous estimate within $1 \sigma$.

Using the empirical polynomial relation between bolometric correction $\mathrm{BC}_{K}$ and $V-J$ in Mann et al. (2015), we find $\mathrm{BC}_{K}$ to be $2.60 \pm 0.13 \mathrm{mag}$. Thus, we derive a bolometric magnitude $M_{\mathrm{bol}}=8.02 \pm 0.13 \mathrm{mag}$, leading to a bolometric luminosity of $L_{*}=$ $0.049 \pm 0.005 L_{\odot}$. To estimate the stellar effective temperature of TOI-530, we first take use of the Stefan-Boltzmann law. Coupled with the aforementioned stellar radius and bolometric luminosity we derived, we get $T_{\text {eff }}=3666 \pm 146 \mathrm{~K}$. As an independent check, we then obtain $T_{\text {eff }}$ following the empirical relation reported by Mann et al. (2015) and we find $T_{\text {eff }}=3650 \pm 100 \mathrm{~K}$. Both estimations agree well with the result $T_{\text {eff }}=3663 \pm 124 \mathrm{~K}$ from Pecaut \& Mamajek (2013).

Finally, we evaluate that TOI-530 has a mass of $M_{*}=$ $0.53 \pm 0.01 \mathrm{M}_{\odot}$ using equation (2) in Mann et al. (2019) according to the $M_{*}-M_{K}$ relation. This is consistent with the value $M_{*}=$ $0.52 \pm 0.03 \mathrm{M}_{\odot}$ given by the EB-based empirical relation of Torres, Andersen \& Giménez (2010).

As an independent check, we carry out an analysis of the broadband spectral energy distribution (SED) together with the Gaia EDR3 parallax in order to determine an independent, empirical measurement of the stellar radius, following the procedures described in Stassun \& Torres (2016), Stassun, Collins \& Gaudi (2017), and Stassun et al. (2018a). We pull the $J H K_{\mathrm{S}}$ magnitudes from $2 M A S S$ (Cutri et al. 2003; Skrutskie et al. 2006), the W1-W3 magnitudes from WISE (Wright et al. 2010), the grizy magnitudes from PanSTARRS (Magnier et al. 2013), and three Gaia magnitudes $G, G_{\mathrm{BP}}$, and $G_{\mathrm{RP}}$ (Gaia Collaboration et al. 2021). Together, the available photometry spans the full stellar SED over the wavelength range 0.4-10 $\mu \mathrm{m}$ (see Fig. 6).

We perform a fit using NextGen stellar atmosphere models, with the $T_{\text {eff }}, \log g$, and $[\mathrm{Fe} / \mathrm{H}]$ taken from the spectroscopic analysis. The remaining parameter is the extinction $\left(A_{V}\right)$, which we limit to the full line-of-sight extinction from the dust maps of Schlegel, Finkbeiner \& Davis (1998). The resulting fit is shown in Fig. 6 with a reduced $\chi^{2}$ of 1.6 and best-fitting extinction of $A_{V}=0.00_{-0.00}^{+0.03}$. Integrating the model SED gives the bolometric flux at Earth of $F_{\text {bol }}=7.009 \pm 0.081 \times 10^{-11} \mathrm{erg} \mathrm{s}^{-1} \mathrm{~cm}^{-2}$. Taking the $F_{\text {bol }}$ and $T_{\text {eff }}$ together with the Gaia parallax, with no adjustment for systematic parallax offset (see, e.g. Stassun \& Torres 2021), gives the stellar radius as $R_{*}=0.547 \pm 0.030 R_{\odot}$.

Combining all the results above, we adopt the weighted-mean values of effective temperature $T_{\text {eff }}$, stellar radius $R_{*}$, and stellar mass $M_{*}$ as listed in Table 3.

To identify the Galactic population membership of TOI-530, we first calculate the three-dimensional space motion with respect to the LSR based on Johnson \& Soderblom (1987). We adopt the astrometric values $\left(\varpi, \mu_{\alpha}, \mu_{\delta}\right)$ from Gaia EDR3 and the spectro-

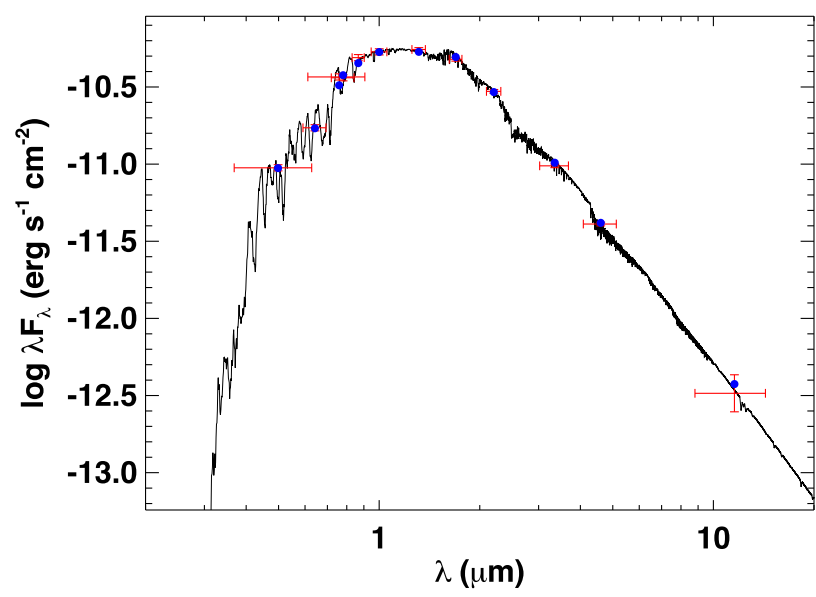

Figure 6. The best SED fit for TOI-530. Red symbols represent the observed photometric measurements, where the horizontal bars represent the effective width of the passband. Blue symbols are the model fluxes from the best-fitting NextGen atmosphere model (black).

Table 3. Basic information of TOI-530.

\begin{tabular}{|c|c|c|}
\hline Parameter & Value & \\
\hline \multicolumn{3}{|l|}{ Main identifiers } \\
\hline TOI & 530 & \\
\hline TIC & 387690507 & \\
\hline Gaia ID & 3353218995355814656 & \\
\hline \multicolumn{3}{|c|}{ Equatorial coordinates } \\
\hline RA (J2015.5) & 06:53:39.08 & \\
\hline Dec. (J2015.5) & $12: 52: 53.68$ & \\
\hline \multicolumn{3}{|c|}{ Photometric properties } \\
\hline TESS (mag) & $13.5287 \pm 0.0076$ & TIC V8 ${ }^{a}$ \\
\hline Gaia (mag) & $14.6217 \pm 0.0006$ & Gaia $\mathrm{EDR}^{b}$ \\
\hline Gaia BP (mag) & $15.814 \pm 0.004$ & Gaia EDR3 \\
\hline Gaia RP (mag) & $13.538 \pm 0.002$ & Gaia EDR3 \\
\hline$B$ (mag) & $16.708 \pm 0.044$ & APASS \\
\hline$V(\mathrm{mag})$ & $15.403 \pm 0.136$ & APASS \\
\hline$J$ (mag) & $12.112 \pm 0.023$ & 2MASS \\
\hline$H$ (mag) & $11.468 \pm 0.030$ & 2MASS \\
\hline$K(\mathrm{mag})$ & $11.238 \pm 0.020$ & 2MASS \\
\hline WISE1 (mag) & $11.124 \pm 0.023$ & WISE \\
\hline WISE2 (mag) & $11.087 \pm 0.020$ & WISE \\
\hline WISE3 (mag) & $10.907 \pm 0.139$ & WISE \\
\hline WISE4 (mag) & $8.735 \pm 0.429$ & WISE \\
\hline \multicolumn{3}{|c|}{ Astrometric properties } \\
\hline$\varpi$ (mas) & $6.77 \pm 0.02$ & Gaia EDR3 \\
\hline$\mu_{\alpha}\left(\operatorname{mas~yr}^{-1}\right)$ & $13.62 \pm 0.03$ & Gaia EDR3 \\
\hline$\mu_{\delta}\left({\left.\operatorname{mas~} \mathrm{yr}^{-1}\right)}^{\prime}\right.$ & $-62.52 \pm 0.02$ & Gaia EDR3 \\
\hline $\mathrm{RV}\left(\mathrm{km} \mathrm{s}^{-1}\right)$ & $-25.93 \pm 2.00$ & This work \\
\hline \multicolumn{3}{|c|}{ Derived parameters } \\
\hline Distance (pc) & $147.7 \pm 0.6$ & This work \\
\hline$U_{\mathrm{LSR}}\left(\mathrm{km} \mathrm{s}^{-1}\right)$ & $48.99 \pm 4.59$ & This work \\
\hline$V_{\mathrm{LSR}}\left(\mathrm{km} \mathrm{s}^{-1}\right)$ & $-20.10 \pm 1.92$ & This work \\
\hline$W_{\mathrm{LSR}}\left(\mathrm{km} \mathrm{s}^{-1}\right)$ & $-6.73 \pm 0.56$ & This work \\
\hline$M_{*}\left(\mathrm{M}_{\odot}\right)$ & $0.53 \pm 0.02$ & This work \\
\hline$R_{*}\left(R_{\odot}\right)$ & $0.54 \pm 0.03$ & This work \\
\hline$\rho_{*}\left(\mathrm{~g} \mathrm{~cm}^{-3}\right)$ & $4.74 \pm 1.11$ & This work \\
\hline $\log g_{*}(\mathrm{cgs})$ & $4.70 \pm 0.03$ & This work \\
\hline$L_{*}\left(L_{\odot}\right)$ & $0.049 \pm 0.005$ & This work \\
\hline$T_{\text {eff }}(\mathrm{K})$ & $3659 \pm 120$ & This work \\
\hline$[\mathrm{Fe} / \mathrm{H}]$ & $0.376 \pm 0.095$ & This work \\
\hline$[\mathrm{M} / \mathrm{H}]$ & $0.218 \pm 0.092$ & This work \\
\hline
\end{tabular}

${ }^{a}$ Stassun et al. (2018b, 2019).

${ }^{b}$ Gaia Collaboration et al. (2021). 

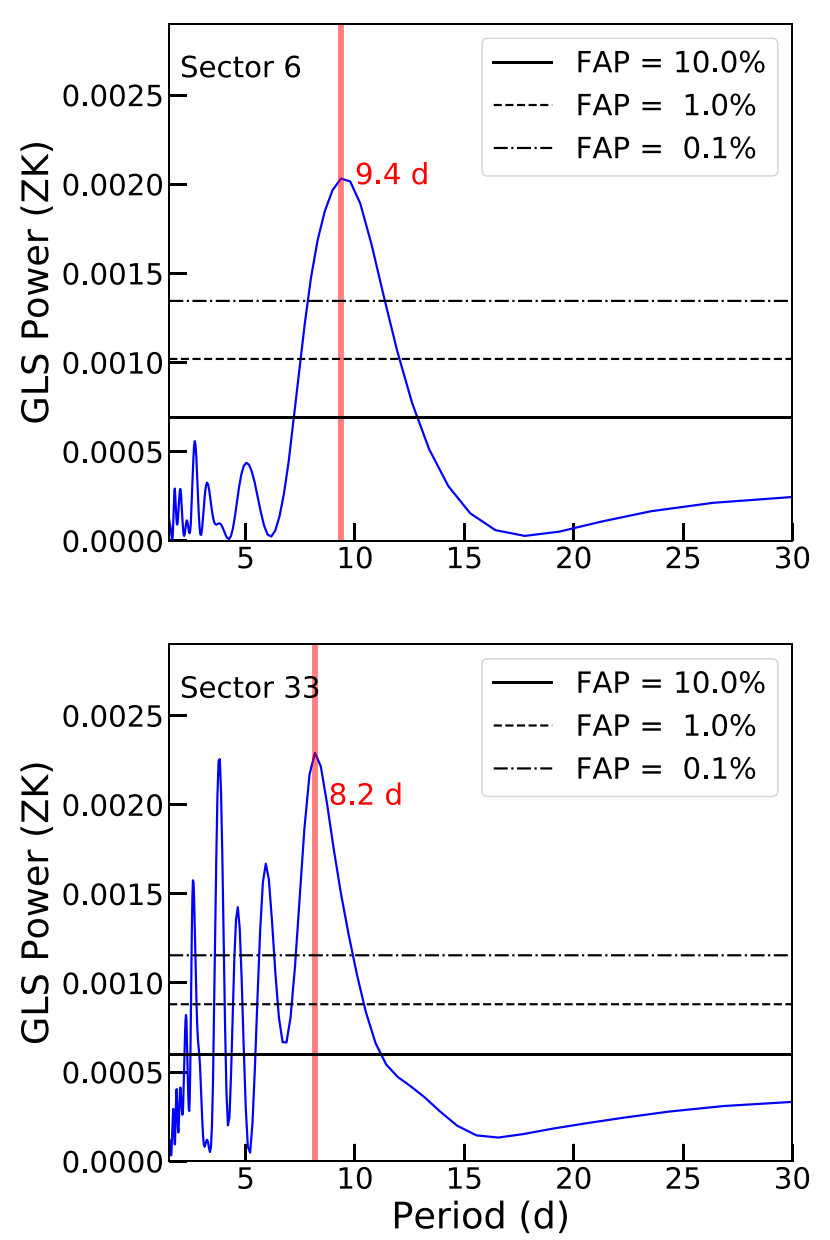

Figure 7. Generalized Lomb-Scargle periodograms of the TESS PDCSAP photometry from two sectors. The theoretical FAP levels of 10, 1, and 0.1 percent are marked as horizontal solid, dashed, and dot-dashed lines. The vertical red lines mark the maximum peaks of the periodograms.

scopically determined systemic RV from the SpeX spectrum, and we find $U_{\mathrm{LSR}}=48.99 \pm 4.59 \mathrm{~km} \mathrm{~s}^{-1}, V_{\mathrm{LSR}}=-20.10 \pm 1.92 \mathrm{~km} \mathrm{~s}^{-1}$, $W_{\mathrm{LSR}}=-6.73 \pm 0.56 \mathrm{~km} \mathrm{~s}^{-1}$. Following the procedure described in Bensby, Feltzing \& Lundström (2003), we compute the relative probability $P_{\text {thick }} / P_{\text {thin }}=0.02$ of TOI-530 to be in the thick and thin discs by taking use of the recent kinematic values from Bensby, Feltzing \& Oey (2014), indicating that TOI-530 belongs to the thin-disc population. We further integrate the stellar orbit with the 'MWPotential2014' Galactic potential using galpy (Bovy 2015) following Gan et al. (2020), and we estimate that the maximal height $Z_{\max }$ of TOI-530 above the Galactic plane is about $109 \mathrm{pc}$, which agrees with our thin-disc conclusion.

We finally perform a frequency analysis on the TESS PDCSAP photometry after masking the known in-transit data using the generalized Lomb-Scargle periodogram (Zechmeister \& Kürster 2009) to look for stellar activity signals. We find a peak at around $9.4 \mathrm{~d}$ in the TESS Sector 6 data (see Fig. 7), which may be attributed to stellar rotation. However, this periodic signal is not significant in the generalized Lomb-Scargle periodogram of the TESS photometry taken in the extended mission. We further analyse the ground-based long-term photometry from the Zwicky Transient Facility (ZTF; Masci et al. 2019). ZTF took a total of 273 exposures for TOI530, which spanned $1036 \mathrm{~d}$. We clip outliers above the $3 \sigma$ level and 242 measurements are left. However, we find that the 9.4-d signal does not show up in the corresponding generalized LombScargle periodogram, either. Additionally, Newton et al. (2018) show a typical rotational period of $\sim 40 \mathrm{~d}$ for a $0.5-\mathrm{M}_{\odot}$ star. We thus conclude that the 9.4-d signal is probably not associated with stellar rotation. Future TESS data to be obtained will allow better identification of the correct rotation period of this target.

\section{ANALYSIS AND RESULTS}

\subsection{Photometric analysis}

\subsubsection{TESS only}

We first model the detrended TESS only photometry by utilizing the juliet package (Espinoza, Kossakowski \& Brahm 2019), which employs batman to build the transit model (Kreidberg 2015). Dynamic nested sampling is applied in juliet to determine the posterior estimates of system parameters using the publicly available package dynesty (Higson et al. 2019; Speagle 2020).

We set uninformative uniform priors on both the transit epoch $\left(T_{0}\right)$ and the orbital period $\left(P_{\mathrm{b}}\right)$, centred on the optimized value obtained from the TLS analysis. Following the approach described in Espinoza (2018), instead of directly fitting for the radius ratio ( $\left.p=R_{\mathrm{p}} / R_{*}\right)$ and the impact parameter $\left(b=a / R_{*} \cos i\right)$, we apply the new parametrizations $r_{1}$ and $r_{2}$ to sample points, for which we impose uniform priors between 0 and 1 . This new parametrization allows us to only sample physically meaningful values of a transiting system with $0<b<1+p$, which reduces the computational cost. We adopt a quadratic limb-darkening law for the TESS photometry, where we place a uniform prior on both coefficients $\left(q_{1}\right.$ and $q_{2}$, Kipping 2013). Since photometric-only data weakly constrain the orbital eccentricity, we fix $e$ at zero and include a non-informative $\log$-uniform prior on stellar density. We fit an extra flux jitter term to account for additional systematics. As the TESS PDCSAP light curve has already been corrected for the light dilution, we fix the dilution factors $D$ to 1 . Table A1 summarizes the prior settings we adopt as well as the best-fitting value of each parameter. We then rerun the photometry-only fit with free $e$ and $w$ to examine potential evidence of eccentricity by comparing the Bayesian model log-evidence $(\ln Z)$ difference between the circular and eccentric orbit models calculated using the dynesty package. Generally, we consider a model is strongly favoured than another if $\Delta \ln Z>5$ (Trotta 2008). We find that the circular orbit model is slightly preferred with a Bayesian evidence improvement of $\Delta \ln Z=\ln Z_{\text {Circular }}-\ln Z_{\text {Keplerian }}=2.8$. We thus conclude that there is no evidence of orbital eccentricity in the TESS time-series data. We use the posteriors from the circular orbit fit as a prior to detrend all ground-based photometric data (see the next section).

\subsubsection{Ground-based photometric data}

Since all of the eight ground light curves only covered partial transits, the way of detrending generally correlates with the final modelling results. Therefore, we decide to independently detrend all ground photometry in a uniform way using Gaussian processes. As there are no obvious quasi-periodic oscillations existing in data from different facilities, we choose the Matérn-3/2 kernel, formulated as

$k_{i, j}(\tau)=\sigma^{2}\left(1+\frac{\sqrt{3} \tau}{\rho}\right) \exp \left(\frac{\sqrt{3} \tau}{\rho}\right)$, 
where $\tau$ is the time-lag, and $\sigma$ and $\rho$ are the covariance amplitude and the correlation time-scale of the GP, respectively. Taking the posteriors from the previous TESS only fit into account, we put a constraint on the priors to optimize the sampling and reduce the computational time cost. We list our priors in Table A2 and show the raw and detrended ground light curves in Fig. 3.

\subsection{RV-only modelling}

We carry out a preliminary RV-only fit using juliet, which utilizes the radvel package to build the Keplerian model (Fulton et al. 2018). In order to reduce the potential errors induced by the orbital period and timing, we fix $P_{b}$ and $T_{0, b}$ at the best-fitting transit ephemeris derived from the previous TESS only fit. Due to the limited number of RV points and our previous insignificant detection of eccentricity (see Section 4.1.1), we fit a circular orbit model with $e$ fixed at zero. Since our RV observations show a clear linear trend, we take the RV slope $\dot{\gamma}$ into consideration in the modelling. Thus the remaining degrees of freedom are the RV semi-amplitude $K_{\mathrm{b}}$, the linear slope $\dot{\gamma}$, the systemic velocity $\mu$ and the extra jitter term $\sigma$, which is used to account for the additional white noise. We adopt wide uniform priors on $K_{b}$ and $\mu$ but a log-uniform prior on $\sigma$, and we allow $\dot{\gamma}$ to vary uniformly between 0 and $100 \mathrm{~m} \mathrm{~s}^{-1} \mathrm{~d}^{-1}$. Our model reveals that the SPIRou RVs have a semi-amplitude of $K=$ $61.3 \pm 12.4 \mathrm{~m} \mathrm{~s}^{-1}$. Table A3 provides our prior settings and the median value of the posterior of each parameter along with their $1 \sigma$ confidence interval.

We then construct a pure linear RV model to test the robustness of our RV detection above. Compared with the linear model, we find that our circular orbit plus an RV slope model has a $\ln Z$ improvement of $\Delta \ln Z=\ln Z_{\text {Circular }}+$ Slope $-\ln Z_{\text {Linear }}=6.5$, supporting a significant planet detection.

\subsection{Joint RV and transit analysis}

In order to obtain precise transit ephemeris and physical parameters, we finally jointly model the detrended TESS photometry and all ground-based re-processed light curves together with the SPIRou RVs. We adopt the identical priors on planetary and TESS photometry parameters as in Section 4.1.1. While for the ground photometric data, we choose the linear law to parametrize the limb-darkening effect and put a Gaussian prior on the theoretical estimate derived from the LDTK package with a width of 0.1 (Husser et al. 2013; Parviainen \& Aigrain 2015). Similarly, we also fit an extra flux jitter term for each ground instrument to account for additional white noise. As there are less contamination in the ground data, we fix all dilution factors $D$ to 1 . For the SPIRou radial velocities, we adopt the same priors as the circular orbit model in Section 4.2. We find the TOI$530 \mathrm{~b}$ has a mass of $0.37 \pm 0.08 M_{J}$ with a radius of $0.83 \pm 0.05 R_{J}$, which is the typical size of a giant planet without much inflation. We show the phase-folded light curves along with the best-fitting models in Figs 8 and 9. Fig. 10 shows the SPIRou data and the best-fitting RV model. Table 4 summarizes the priors we set in the final joint fit as well as the best-fitting value of each parameter. We list the final derived physical parameters in Table 5.

Since there are a total of five nearby stars of TOI-530 with $T_{\text {mag }}<$ 15.5 located within 1 arcmin and the light from the brightest star among them (Gaia DR2 3353218784898973312, $T_{\text {mag }}=11.0$; star 5 on the right-hand panel of Fig. 1) is expected to have a significant contribution of the contamination flux in the photometric aperture due to the large TESS pixel scale (21 arcsec pixel $\left.{ }^{-1}\right)$, we rerun the joint fit to examine whether additional dilution correction is needed.
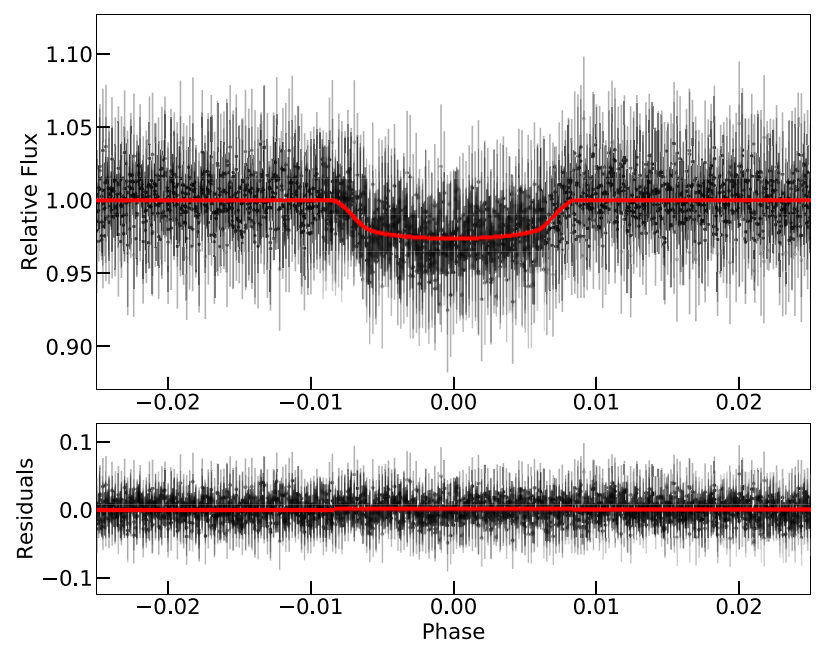

Figure 8. Top panel: phase-folded TESS photometry of TOI-530. The red solid line represents the median posterior model. Bottom panel: the residuals of the TESS data after subtracting the best-fitting transit model.
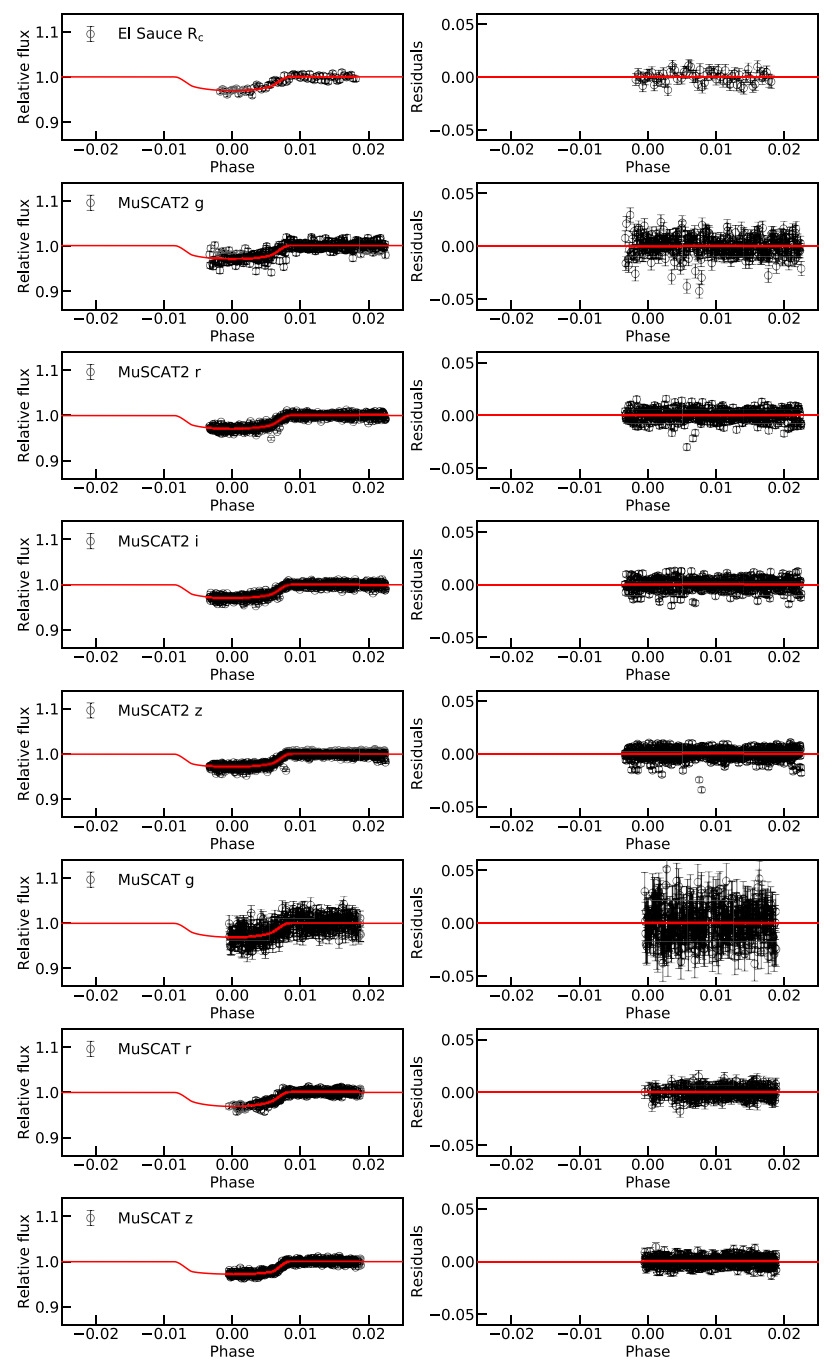

Figure 9. Left-hand panels show unbinned phase-folded follow-up transit light curves of TOI-530. The instrument and observational band information is presented at the top left-hand of each panel. Our best-fitting models are shown as red solid lines. The residuals are shown on the right-hand panels. 

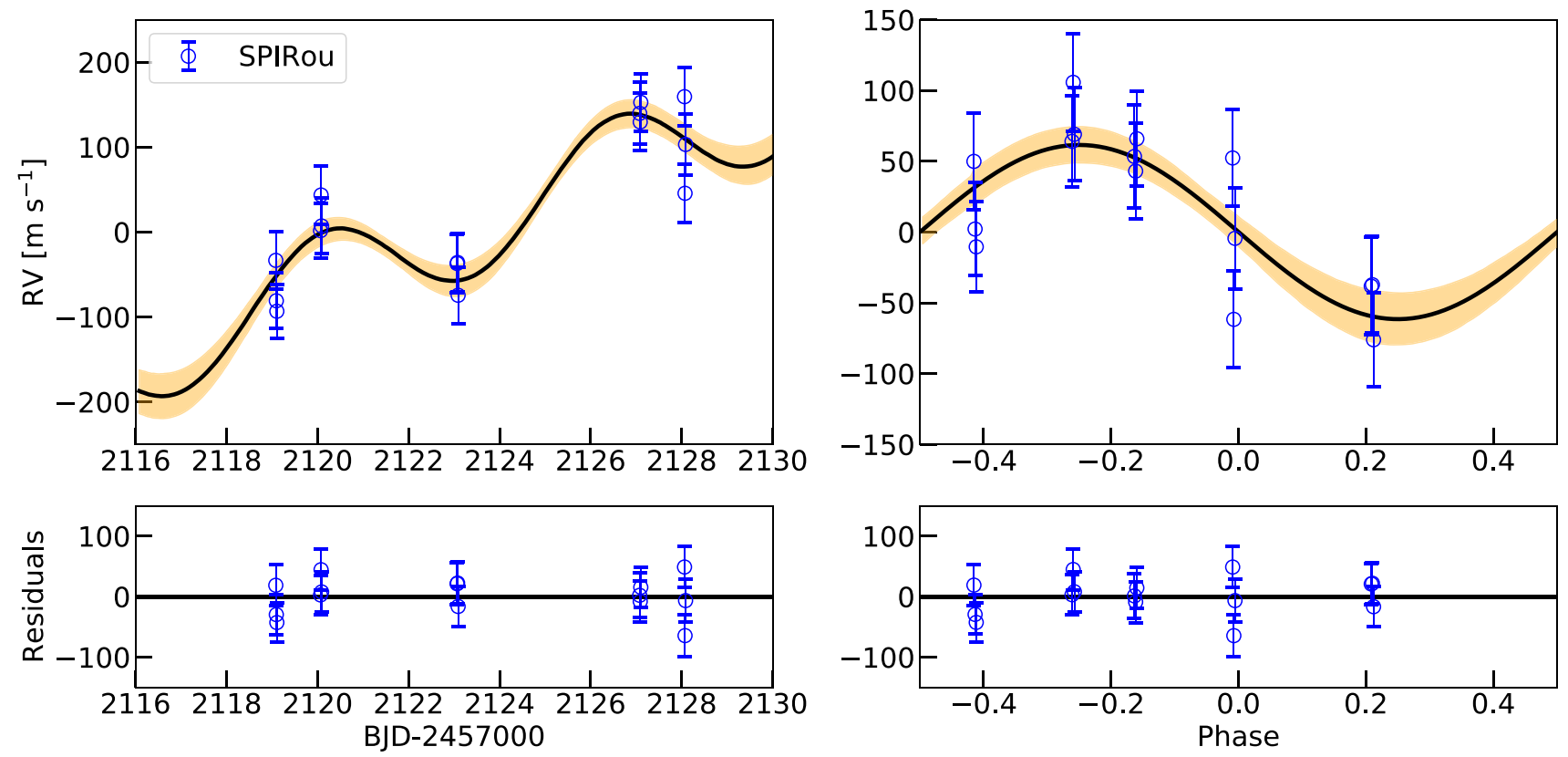

Figure 10. Left-hand panel: The SPIRou RVs of TOI-530 as a function of time along with the best-fitting circular orbit model from the photometry + RV joint analysis shown as a black solid line. The error bars are the quadrature sum of the instrument jitter term and the measurement uncertainties for all RVs. The orange shaded region represents the $1 \sigma$ confidence interval of the model. Right-hand panel: The corresponding phased-folded SPIRou RV data after subtracting the RV slope. Residuals are plotted below.

We set a Gaussian prior on the TESS dilution factor $D_{\mathrm{TESS}}$, centred at 1 with a $1 \sigma$ width of 0.1 , and keep the left prior settings the same as above. We obtain $D_{\mathrm{TESS}}=0.97 \pm 0.03$ and a radius ratio of $R_{\mathrm{p}} / R_{*}=$ $0.156 \pm 0.001$, consistent with the result without considering light correction.

\section{DISCUSSION}

\subsection{A lack of hot massive giant planets around M-dwarfs?}

Fig. 11 shows the planet-to-star mass ratio $(q)$ as a function of separation distance $(a)$ of all giant planets $\left(0.3 M_{J} \leq M_{p}<13.6 M_{J}\right)$ around M-dwarfs detected by different methods. Regarding the microlensing sample, since most lens systems are still blended with their sources, it is hard to determine the spectral type of the host star in the lens system. ${ }^{5}$ Thus we simply set a host-mass threshold between 0.08 and $0.65 \mathrm{M}_{\odot}$, and we filter out targets that meet the mass cut. While for the other three, we pick out the sample mainly based on the spectral information. We only consider the mass ratio here because most microlensing light-curve analyses do not provide the masses of the host and the planet, although the planet-to-host mass ratio, $q$, is well determined (Mao \& Paczynski 1991; Gould \& Loeb 1992). To measure the mass of microlensing planet, one needs two observables (Zang et al. 2020), but most microlensing planets do not have them, and thus a Bayesian analysis is needed to estimate the host mass, which has a typical $1 \sigma$ uncertainty of $\sim 0.3 \mathrm{M}_{\odot}$. Thus, it is challenging to classify microlensing planets according to different types of host stars. However, several microlensing detections with

${ }^{5}$ Even if the lens and their sources have separated after sufficient long time due to the proper motion, it is still difficult as the host stars of the lens systems are very faint (typically $V \sim 25 \mathrm{mag}$ ). unambiguous mass measurements demonstrate that gaint planets orbiting M-dwarfs are common (e.g. Bennett et al. 2020).

Four giant planets identified by direct imaging that are far from their host M-dwarfs are located at the high-mass-ratio region. This is likely caused by observational biases as the imaging method has difficulty to detect low-mass Jupiters with $M_{\mathrm{p}}$ around $1 M_{\mathrm{J}}$ (all of these four planets have $M_{\mathrm{p}} \gtrsim 10 M_{\mathrm{J}}$ ). Microlensing, however, is sensitive to all kinds of widely separated planets with masses ranging from super-Jupiter down to Earth (e.g. Zang et al. 2021). A total of 55 giant planets harboured by M-dwarfs have been discovered with projected separation distance $a_{\perp} \gtrsim 1$ au. ${ }^{6}$ There is a wide mass ratio distribution of those microlensing systems, most of which have $q \lesssim$ $10^{-2}$, indicating that cold Jupiters around M-dwarfs are possibly common and diverse.

A similar trend can also been seen in the RV-only sample whose separation distances are between 0.1 and $10 \mathrm{au}$, although RV can only determine the minimum mass ratio $q_{\min }$ for those non-transiting systems. Currently, there are no RV-only giant planets with $q_{\text {min }} \geq$ $10^{-2}$ that have been detected around M-dwarfs, which is likely due to observational biases as follows. Unlike microlensing, which is not limited by the lens flux, determining the companion mass spectroscopically requires central stars to be relatively bright (typically $V<$ $13 \mathrm{mag}$ ). Thus the RV-only sample may miss giant planets around faint late-type M-dwarfs, which have higher $q_{\min }$ compared with equivalent planets around early-type M-dwarfs. For massive earlytype M-dwarfs, however, some of their companions within that mass ratio range should belong to brown dwarfs, which are not included here. Furthermore, no giant planets have been detected within $0.1 \mathrm{au}$ of their host M-dwarfs from RV-only surveys. This phenomenon can be attributed to the RV observational strategy. Most RV surveys

${ }^{6}$ The solutions of 12 microlensing systems have the so-called close-wide degeneracy, shown in pairs as translucent black squares in Fig. 11. 
Table 4. Prior settings and the best-fitting values along with the 68 percent credibility intervals in the final joint fit for TOI-530. $\mathcal{N}\left(\mu, \sigma^{2}\right)$ means a normal prior with mean $\mu$ and standard deviation $\sigma \cdot \mathcal{U}(\mathrm{a}, \mathrm{b})$ stands for a uniform prior ranging from a to $\mathrm{b} . \mathcal{J}(\mathrm{a}, \mathrm{b})$ stands for a Jeffrey's prior ranging from a to $\mathrm{b}$.

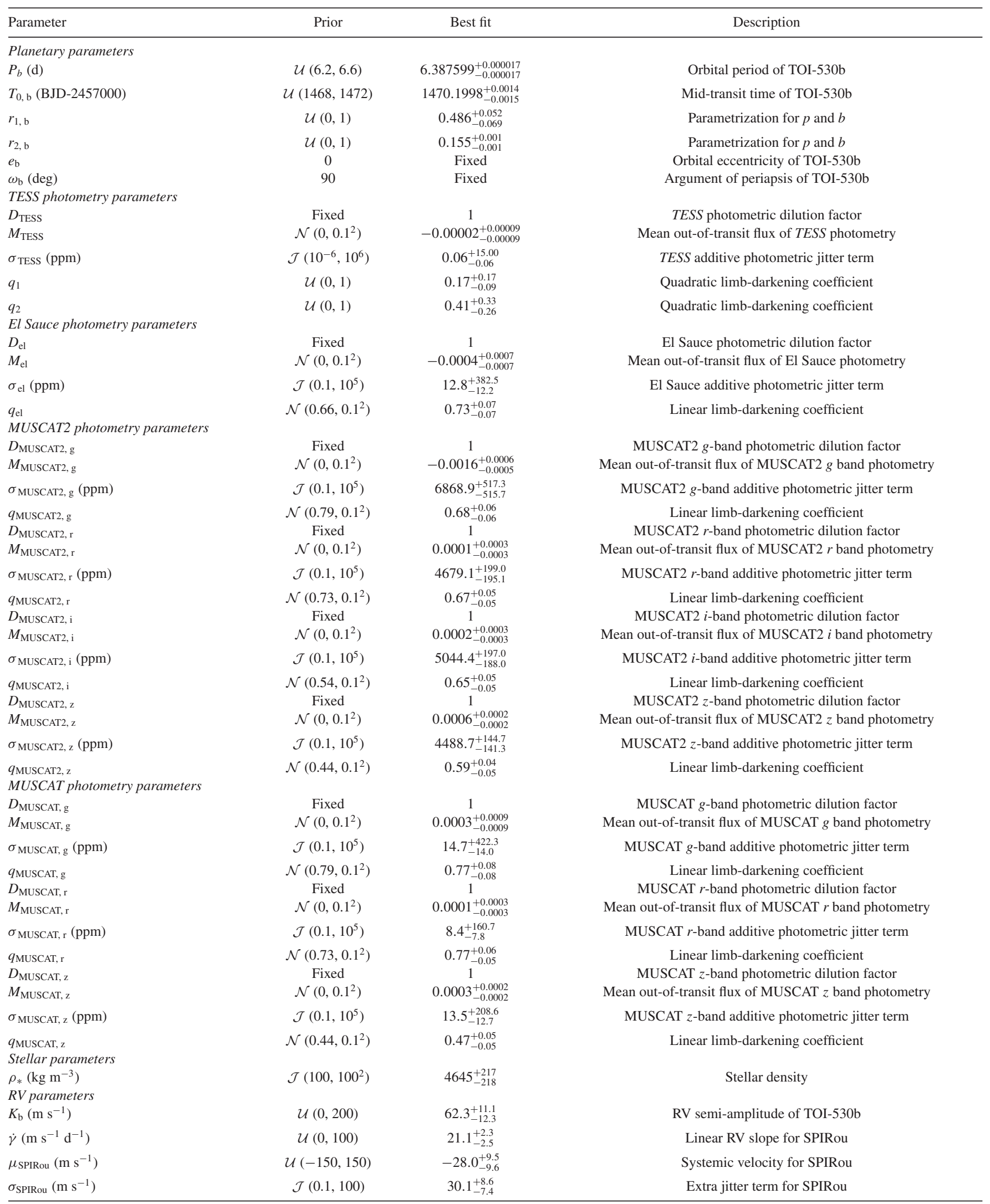


Table 5. Derived physical parameters from the final joint fit for TOI-530.

\begin{tabular}{lcl}
\hline Parameter & Best fit & \multicolumn{1}{c}{ Description } \\
\hline$R_{\mathrm{p}} / R_{*}$ & $0.155_{-0.002}^{+0.002}$ & Planet radius in units of stellar radius \\
$R_{\mathrm{p}}\left(R_{J}\right)$ & $0.83_{-0.06}^{+0.06}$ & Planet radius \\
$M_{\mathrm{P}}\left(M_{J}\right)$ & $0.37_{-0.07}^{+0.08}$ & Planet mass \\
$\rho_{\mathrm{p}}\left(\mathrm{g} \mathrm{cm}^{-3}\right)$ & $0.70_{-0.27}^{+0.38}$ & Planet density \\
$b$ & $0.23_{-0.10}^{+0.07}$ & Impact parameter \\
$a / R_{*}$ & $21.56_{-0.34}^{+0.33}$ & Semimajor axis in units of stellar radii \\
$a(\mathrm{au})$ & $0.054_{-0.004}^{+0.004}$ & Semimajor axis \\
$i\left(^{\circ}\right)$ & $89.4_{-0.2}^{+0.3}$ & Inclination angle \\
$T_{\mathrm{eq}}^{a}(\mathrm{~K})$ & $557_{-22}^{+23}$ & Equilibrium temperature \\
\hline
\end{tabular}

${ }^{a}$ We assume there is no heat distribution between the dayside and nightside, and that the albedo is zero.

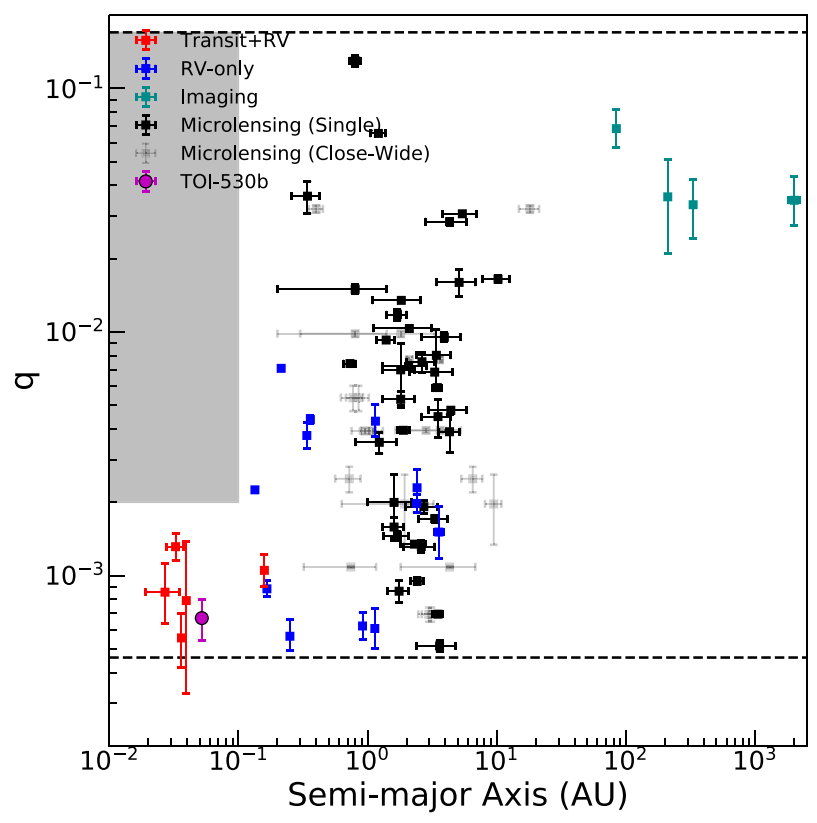

Figure 11. Planet-to-star mass ratio of all giant planets around M-dwarfs as a function of semi-major axis. Different colours represent planets detected by different methods. The two horizontal black dashed lines represent the selection threshold of our sample. The upper limit is $q=13.6 M_{J} / 0.08 \mathrm{M}_{\odot}=$ 0.17 , while the lower limit corresponds to $q=0.3 M_{J} / 0.65 \mathrm{M}_{\odot}=4.6 \times 10^{-4}$. TOI-530b is marked as a magenta circle. The grey shaded region represents a possible paucity of hot massive giant planets around M-dwarfs.

focus on bright nearby M-dwarfs and the total sample size is small (roughly $\sim 200$ ). Thus it is reasonable to find none RV-only giant planets in this region given the low occurrence rate of hot Jupiter $(\sim 0.5$ per cent, Fressin et al. 2013; Petigura et al. 2018; Zhou et al. 2019). Therefore, deep transit surveys play a crucial role in detecting such candidates as they are sensitive to planets with small semimajor axis and large mass ratio, which also more likely to have a large planet-to-star radius ratio.

Interestingly, all five known transiting giant planet systems and TOI-530b turn out to have small mass ratio $q \sim 10^{-3}$ and there is a possible dearth at the region with $q>2 \times 10^{-3}$ and $a<0.1$ au (see the shaded region in Fig. 11). Part of that may result from the flux-limit problem above (for $q \geq 10^{-2}$ ). We note that this deficiency feature may reflect a more fundamental link to the planet formation theory.
Recent work from Liu et al. (2019) constructed a pebble-driven planet population synthesis model, and their simulation results suggest that gas giants may mainly form when the central stars are more massive than $0.3 \mathrm{M}_{\odot}$ (see fig. 7 in Liu et al. 2019). This is because planets stop increasing their core masses when they reach the pebble isolation mass $M_{\text {iso }}$, which is proportional to the stellar mass as $M_{\text {iso }} \propto M_{*}^{4 / 3}$. Following gas accretion on to planets with small $M_{\text {iso }}$ is limited due to a slow Kelvin-Helmholtz contraction. Thus, they would stop before the runaway gas accretion and be left as rock- or ice-dominated planets with tiny atmospheres. If this is the case, we then expect few giant planets with relatively high mass ratio above $10^{-3}$ when their host masses are below $0.3 \mathrm{M}_{\odot}$. Note that the known 'brown dwarf desert' ( $35 \leq M \sin i \leq 55 M_{J}$ and orbital periods under 100 days), studied by Ma \& Ge (2014) using all available data of close brown dwarfs around solar-type stars, is also located in this region with $q \gtrsim 0.05$. This lower limit is estimated based on the lower limit of the brown dwarf desert $35 M_{J}$ and the typical mass upper limit of M-dwarfs $0.65 \mathrm{M}_{\odot}$. However, it is still unclear whether the deficiency between $2 \times 10^{-3}$ and $10^{-2}$ in mass ratio is physical. Compared with the known planets (red squares plus TOI-530b in Fig. 11), the transit method is, in principle, more sensitive to giant planets with a larger mass ratio (i.e. larger radius ratio) located in this deficiency region. Additionally, if detected by transit survey, planet candidates within this parameter space range should be easily confirmed by the RV method. Due to the lack of transiting giant planets around M-dwarfs, we cannot draw any conclusions yet. Hopefully, the TESS QLP Faint Star $(10.5<$ $T<13.5 \mathrm{mag}$ ) Search could provide more such systems and check if this depletion feature is real (Kunimoto et al., in preparation).

\subsection{Metallicity dependence}

Although the core accretion theory (Pollack et al. 1996) has predicted rare giant planets around M-dwarfs due to their low protoplanetary disc mass as $M_{\text {disc }}$ linearly scales with the stellar mass $M_{*}$ (Andrews et al. 2013), this defect may be compensated if the parent star is metal rich, which could theoretically supply more solid material used for accretion. Alternatively, gravitational instability (GI) could also form giant planets around M-dwarfs (Boss 2006), though simulation work from Cai et al. (2006) suggested that GI is unlikely the major mechanism that produce most observed planets. Under the GI hypothesis, we expect that there would not exist a strong dependence between giant planet formation and host metallicity and that even stars with relatively low metallicity should harbour gas giants (Boss 2002).

In order to investigate the metallicity dependence observationally, we retrieve a list of solar-type stars (simply selected based on $0.90<$ $\left.M_{*}<1.06 \mathrm{M}_{\odot}\right)$ hosting giant planets from the NASA Exoplanet Archive (Akeson et al. 2013). We find a total of 88 transiting and 102 RV-only systems. Most transiting giant planets are hot with semimajor axis $a \lesssim 0.1 \mathrm{AU}$, while the majority of RV-only planets are cold with semimajor axes beyond 0.1 au. Fig. 12 illustrates the metallicity distribution of their hosts, indicating that hot and cold giant planets do not present much difference in $[\mathrm{Fe} / \mathrm{H}]$ preference around solar-type stars (see the translucent points in Fig. 12). The weighted-mean metallicity of both transiting and RV-only giant planet central stars is above the solar value but almost the same: 0.12 and 0.14 , respectively. This is consistent with the conclusions from early work suggesting that the frequency of giant planets increases with stellar metallicity (Santos, Israelian \& Mayor 2004; Fischer \& Valenti 2005; Sousa et al. 2011). However, it is not the same for gas giants around M-dwarfs.

Among all giant planets around M-dwarfs, we only find four transiting and nine RV-only systems that have metallicity measurements 


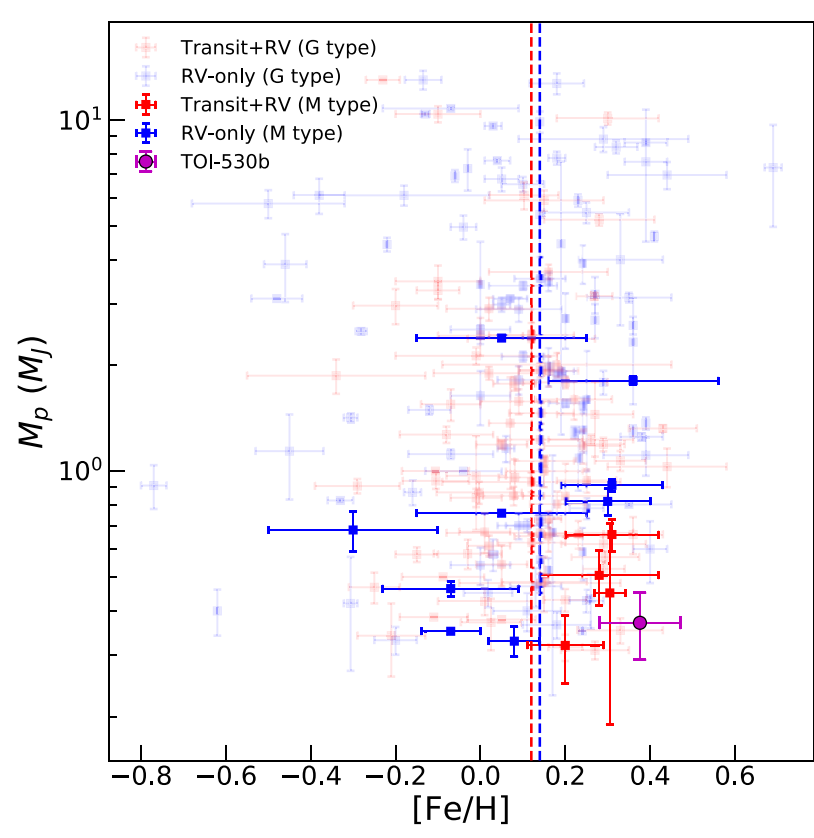

Figure 12. Planet mass $M_{\mathrm{p}}$ versus host star metallicity. Different colours represent planets detected by different methods (transit/RV). The red and blue dashed lines are the weighted-mean metallicity of solar-type stars hosting giant planets detected by transit and RV-only observations. TOI-530b is marked as a magenta circle. All hot giant planet M-dwarf hosts tend to have a higher metallicity than G dwarf hosts (see Section 5.2).

in the literature. For cold (RV-only) giant planets, the metallicities of their M-dwarf hosts are distributed on both sides of the median value 0.14 (the blue dashed line in Fig. 12) seen for the solar-type stars, though the uncertainties are large. Some of them are formed around metal-poor M stars (e.g. GJ 832b, Bailey et al. 2009). This is plausibly in agreement with the previous finding that clump formation is fairly insensitive to the metallicity of the parent star (Boss 2002), implying that part of the formation of cold giant planets around M-dwarfs may take place through GI. Indeed, the recently detected planetary system GJ $3512 b$, whose host star has a solar metallicity $-0.07 \pm 0.16$, favours the GI formation scenario (Morales et al. 2019).

However, the host stars of four known transiting giant planets together with TOI-530 tend to be metal rich, all of which have $[\mathrm{Fe} / \mathrm{H}]$ higher than the aforementioned median value 0.12 (the red dashed line in Fig. 12). It indicates that the formation of hot (transiting) giant planets around M-dwarfs may have a strong dependence on the host metallicity as predicted by the core accretion theory. This is consistent with the recent findings from Maldonado et al. (2020) (see the left-hand panel of their fig. 14), which reveals a correlation between the metallicities of M-dwarfs and their probability of hosting giant planets. Compared with hot giant planets around solar-type stars, the formation of hot giant planets around M-dwarfs possibly requires higher host metallicity, though the number of detections is probably too small to confirm this claim. To examine the statistical significance of this feature, we carry out a Kolmogorov-Smirnov (K-S) test. We calculate the K-S statistic between the metallicities of stars in the $\mathrm{G}$ - and M-type transiting sample, which yields a $p$-value of 0.023 . It roughly corresponds to the $2.5 \sigma$ significance level, at which we can reject the null hypothesis that two samples are from the same distribution. We then adopt the bootstrap method to randomly draw distributions from the G-type parent sample and compute the $\mathrm{K}-\mathrm{S}$ statistic between these random distributions and the M-type transiting sample. We repeat this procedure for 10000 times and derive the corresponding $p$ value distribution. We find that 88 per cent of the total random samples have $p$-values less than 0.05 ( $2 \sigma$ level) while only 0.3 percent of them have $p$-values less than 0.003 ( $3 \sigma$ level), indicating a marginal correlation. Future detections of more hot giant planets around M-dwarfs will reveal whether this metallicity preference is significant or not.

\subsection{Prospects for future observations}

Due to the faintness of the host star, high precision RV spectrographs on large telescopes like the red-optical MAROON-X (Seifahrt et al. 2018) or near-infrared instruments like InfrRed Doppler spectrograph (IRD; Kotani et al. 2018) are required to reach sufficient signal-to-noise ratio. The host star is quiet without strong intrinsic stellar activity as there are no significant flux variations in the TESS light curve, making it a suitable target for precise RV follow-up observations. Though a potential 9.4-d modulation signal is shown up in the TESS light curve, this is probably not linked to the stellar rotation given our ZTF results and previous findings from Newton et al. (2018) (see Section 3).

Measuring the stellar obliquity of M-dwarfs hosting hot giant planets could provide clues about their origins and gain insights into their migration history. To probe the potential opportunities to observe the Rossiter-McLaughlin effect (McLaughlin 1924; Rossiter 1924) of TOI-530 and measure the projected angle between the planet orbital and stellar equatorial planes, we estimate the RM semiamplitude of this system as

$A_{\mathrm{RM}} \simeq \frac{2}{3}\left(R_{p} / R_{*}\right)^{2} \sqrt{1-b^{2}} \times v \sin i$,

where $b$ is the impact parameter and $v \sin i$ is the projected stellar equatorial rotation velocity. Taking the best-fitting values from the light curve modelling, assuming a rotational period of $40 \mathrm{~d}$ for a $0.5-\mathrm{M}_{\odot}$ star (see fig. 4 in Newton et al. 2018) with an upper and lower limit of 100 and $30 \mathrm{~d}$, we find $A_{\mathrm{RM}} \sim 16_{-6}^{+4} \mathrm{~m} \mathrm{~s}^{-1}$, making the $\mathrm{RM}$ signal challenging to be detected even with near-infrared RV observations.

Finally, we investigate the atmospheric characterization possibility by calculating the Transmission Spectroscopy Metric (TSM; Kempton et al. 2018) of TOI-530b. We obtain a TSM of $53_{-21}^{+39}$, which is much smaller than the recommended threshold of 90 for high-quality sub-Jovians $\left(4.0<R_{\mathrm{p}}<10 R_{\oplus}\right)$ targets from Kempton et al. (2018). Thus, we conclude that TOI-530b is not a promising target for future atmospheric composition studies.

\section{SUMMARY AND CONCLUSIONS}

In this paper, we present the discovery and characterization of a transiting giant planet TOI-530b around an early, metal-rich Mdwarf. Space and ground photometry as well as SPIRou RVs reveal that TOI-530b has a radius of $0.83 \pm 0.05 R_{J}$ and a mass of $0.37 \pm 0.08 M_{J}$ on a 6.39 -d orbit. Although it is challenging to probe the atmospheric properties of TOI-530b due to the faintness of the host star, TOI-530 is still a suitable target to study the stellar obliquity. Furthermore, we report a potential paucity of hot massive giant planets around M-dwarfs with separation distance smaller than 0.1 au and planet-to-star mass ratio between $2 \times 10^{-3}$ and $10^{-2}$. We also identify a possible correlation between hot giant planet formation and the metallicity of its parent M-dwarf. However, due to the current small sample of such systems, we could not make any firm conclusions in this context. Future near-infrared 
spectroscopic surveys such as SPIRou Legacy Survey-Planet Search (SLS-PS; Moutou et al. 2017; Fouqué et al. 2018) shall remedy this situation.

\section{ACKNOWLEDGEMENTS}

We are grateful to Coel Hellier for the insights regarding the WASP data. We also thank Elisabeth Newton, Robert Wells, Hongjing Yang, and Weicheng Zang for useful discussions. We also thank Elise Furlan for the contributions to the speckle data and Nadine Manset for scheduling the SPIRou observations. This work is partly supported by the National Science Foundation of China (Grant No. 11390372 and 11761131004 to SM and TG). This research uses data obtained through the Telescope Access Program (TAP), which has been funded by the TAP member institutes. This work is partly supported by JSPS KAKENHI Grant Numbers JP17H04574, JP18H05439, 20K14521, JST PRESTO Grant Number JPMJPR1775, and the Astrobiology Center of National Institutes of Natural Sciences (NINS) (Grant Number AB031010). This article is based on observations made with the MuSCAT2 instrument, developed by ABC, at Telescopio Carlos Sánchez operated on the island of Tenerife by the IAC in the Spanish Observatorio del Teide. Some of the observations in the paper made use of the High-Resolution Imaging instrument 'Alopeke obtained under LLP GN-2021A-LP-105. 'Alopeke was funded by the NASA Exoplanet Exploration Program and built at the NASA Ames Research Center by Steve B. Howell, Nic Scott, Elliott P. Horch, and Emmett Quigley. Data were reduced using a software pipeline originally written by Elliott Horch and Mark Everett. 'Alopeke was mounted on the Gemini North telescope of the international Gemini Observatory, a program of NSF's OIR Lab, which is managed by the Association of Universities for Research in Astronomy (AURA) under a cooperative agreement with the National Science Foundation. on behalf of the Gemini partnership: the National Science Foundation (United States), National Research Council (Canada), Agencia Nacional de Investigación y Desarrollo (Chile), Ministerio de Ciencia, Tecnología e Innovación (Argentina), Ministério da Ciência, Tecnologia, Inovações e Comunicações (Brazil), and Korea Astronomy and Space Science Institute (Republic of Korea). Funding for the TESS mission is provided by NASA's Science Mission directorate. We acknowledge the use of TESS public data from pipelines at the TESS Science Office and at the TESS Science Processing Operations Center. Resources supporting this work were provided by the NASA High-End Computing (HEC) Program through the NASA Advanced Supercomputing (NAS) Division at Ames Research Center for the production of the SPOC data products. This research has made use of the Exoplanet Follow-up Observation Program website, which is operated by the California Institute of Technology, under contract with the National Aeronautics and Space Administration under the Exoplanet Exploration Program. This paper includes data collected by the TESS mission, which are publicly available from the Mikulski Archive for Space Telescopes (MAST). This work has made use of data from the European Space Agency (ESA) mission Gaia (https://www.cosmos.esa.int/gaia), processed by the Gaia Data Processing and Analysis Consortium (DPAC, https: //www.cosmos.esa.int/web/gaia/dpac/consortium). Funding for the DPAC has been provided by national institutions, in particular the institutions participating in the Gaia Multilateral Agreement. This work made use of tpfplotter by J. Lillo-Box (publicly available in www.github.com/jlillo/tpfplotter), which also made use of the python packages astropy, lightkurve, matplotlib and numpy.

\section{DATA AVAILABILITY}

This paper includes photometric data collected by the TESS mission and ground instruments, which are publicly available in ExoFOP, at https://exofop.ipac.caltech.edu/tess/target.php?id = 387690507. All spectroscopy data underlying this paper are listed in the text. All of the high-resolution speckle imaging data are available at the NASA exoplanet Archive with no proprietary period.

\section{REFERENCES}

Akeson R. L. et al., 2013, PASP, 125, 989

Albrecht S. et al., 2012, ApJ, 757, 18

Aller A., Lillo-Box J., Jones D., Miranda L. F., Barceló Forteza S., 2020, A\&A, 635, A128

Andrews S. M., Rosenfeld K. A., Kraus A. L., Wilner D. J., 2013, ApJ, 771, 129

Apps K. et al., 2010, PASP, 122, 156

Artigau É. et al., 2014a, in Ramsay S. K., McLean I. S., Takami H., eds, SPIE Conf. Ser. Vol. 9147, Ground-based and Airborne Instrumentation for Astronomy V. SPIE, Bellingham, p. 914715

Artigau É. et al., 2014b, in Peck A. B., Benn C. R., Seaman R. L., eds, SPIE Conf. Ser. Vol. 9149, Observatory Operations: Strategies, Processes, and Systems V. SPIE, Bellingham, p. 914905

Artigau É. et al., 2021, AJ, 162, 144

Baglin A., Auvergne M., Barge P., Deleuil M., Catala C., Michel E., Weiss W., COROT Team, 2006, in Fridlund M., Baglin A., Lochard J., Conroy L., eds, ESA SP-1306, The CoRoT Mission Pre-Launch Status - Stellar Seismology and Planet Finding. ESA, Noordwijk, p. 33

Bailey J., Butler R. P., Tinney C. G., Jones H. R. A., O’Toole S., Carter B. D., Marcy G. W., 2009, ApJ, 690, 743

Bakos G., Noyes R. W., Kovács G., Stanek K. Z., Sasselov D. D., Domsa I., 2004, PASP, 116, 266

Bakos G. Á. et al., 2020, AJ, 159, 267

Barentsen G. et al., 2019, Keplergo/lightkurve: Lightkurve v1.0b29. Zenodo. Available at: https://doi.org/10.5281/zenodo.2565212

Batalha N. E., Lewis N. K., Line M. R., Valenti J., Stevenson K., 2018, ApJ, 856, L34

Bayliss D. et al., 2018, MNRAS, 475, 4467

Bedell M., Hogg D. W., Foreman-Mackey D., Montet B. T., Luger R., 2019, AJ, 158, 164

Bennett D. P. et al., 2020, AJ, 159, 68

Bensby T., Feltzing S., Lundström I., 2003, A\&A, 410, 527

Bensby T., Feltzing S., Oey M. S., 2014, A\&A, 562, A71

Bertaux J. L., Lallement R., Ferron S., Boonne C., Bodichon R., 2014, A\&A, 564, A46

Borucki W. J. et al., 2010, Science, 327, 977

Boss A. P., 2000, ApJ, 536, L101

Boss A. P., 2002, ApJ, 567, L149

Boss A. P., 2006, ApJ, 643, 501

Bovy J., 2015, ApJS, 216, 29

Boyajian T. S., van Belle G., von Braun K., 2014, AJ, 147, 47

Butler R. P., Vogt S. S., Marcy G. W., Fischer D. A., Wright J. T., Henry G. W., Laughlin G., Lissauer J. J., 2004, ApJ, 617, 580

Butler R. P., Johnson J. A., Marcy G. W., Wright J. T., Vogt S. S., Fischer D. A., 2006, PASP, 118, 1685

Cai K., Durisen R. H., Michael S., Boley A. C., Mejía A. C., Pickett M. K., D’Alessio P., 2006, ApJ, 636, L149

Cañas C. I. et al., 2020, AJ, 160, 147

Chazelas B. et al., 2012, in Stepp L. M., Gilmozzi R., Hall H. J., eds, Proc. SPIE Conf. Ser. Vol. 8444, Ground-based and Airborne Telescopes IV. SPIE, Bellingham, p. 84440E

Ciardi D. R., Beichman C. A., Horch E. P., Howell S. B., 2015, ApJ, 805, 16

Cloutier R. et al., 2018, AJ, 155, 93

Collins K. A., Kielkopf J. F., Stassun K. G., Hessman F. V., 2017, AJ, 153, 77

Cushing M. C., Vacca W. D., Rayner J. T., 2004, PASP, 116, 362 
Cushing M. C., Rayner J. T., Vacca W. D., 2005, ApJ, 623, 1115

Cutri R. M. et al., 2003, 2MASS All Sky Catalog of Point Sources, VizieR Online Data Catalog, p. II/246

Diamond-Lowe H., Charbonneau D., Malik M., Kempton E. M. R., Beletsky Y., 2020, AJ, 160, 188

Donati J.-F. et al., 2018, SPIRou: A NIR Spectropolarimeter/High-Precision Velocimeter for the CFHT. Springer, Berlin, p. 107

Donati J. F. et al., 2020, MNRAS, 498, 5684

Dressing C. D., Charbonneau D., 2013, ApJ, 767, 95

Dressing C. D., Charbonneau D., 2015, ApJ, 807, 45

Dressing C. D. et al., 2019, AJ, 158, 87

Espinoza N., 2018, Res. Notes Am. Astron. Soc., 2, 209

Espinoza N., Kossakowski D., Brahm R., 2019, MNRAS, 490, 2262

Fischer D. A., Valenti J., 2005, ApJ, 622, 1102

Foreman-Mackey D., Agol E., Ambikasaran S., Angus R., 2017, AJ, 154, 220

Fouqué P. et al., 2018, MNRAS, 475, 1960

Fressin F. et al., 2013, ApJ, 766, 81

Fukui A. et al., 2011, PASJ, 63, 287

Fulton B. J., Petigura E. A., Blunt S., Sinukoff E., 2018, PASP, 130, 044504

Furlan E. et al., 2017, AJ, 153, 71

Gaia Collaboration et al., 2021, A\&A, 649, A1

Gan T. et al., 2020, AJ, 159, 160

Gilbert E. A. et al., 2020, AJ, 160, 116

Gould A., Loeb A., 1992, ApJ, 396, 104

Guerrero N. M. et al., 2021, ApJS, 254, 39

Hardegree-Ullman K. K., Cushing M. C., Muirhead P. S., Christiansen J. L., 2019, AJ, 158, 75

Hartman J. D. et al., 2015, AJ, 149, 166

Henry T. J., Jao W.-C., Subasavage J. P., Beaulieu T. D., Ianna P. A., Costa E., Méndez R. A., 2006, AJ, 132, 2360

Higson E., Handley W., Hobson M., Lasenby A., 2019, Stat. Comput., 29, 891

Hippke M., Heller R., 2019, A\&A, 623, A39

Howard A. W. et al., 2010, ApJ, 721, 1467

Howell S. B., Everett M. E., Sherry W., Horch E., Ciardi D. R., 2011, AJ, 142,19

Howell S. B. et al., 2014, PASP, 126, 398

Howell S. B., Everett M. E., Horch E. P., Winters J. G., Hirsch L., Nusdeo D., Scott N. J., 2016, ApJ, 829, L2

Howell S. B., Matson R. A., Ciardi D. R., Everett M. E., Livingston J. H., Scott N. J., Horch E. P., Winn J. N., 2021, AJ, 161, 164

Husser T.-O., Wende-von Berg S., Dreizler S., Homeier D., Reiners A., Barman T., Hauschildt P. H., 2013, A\&A, 553, A6

Ida S., Lin D. N. C., 2005, ApJ, 626, 1045

Jenkins J. M., 2002, ApJ, 575, 493

Jenkins J. M. et al., 2016, in Chiozzi G., Guzman J. C., eds, SPIE Conf. Ser. Vol. 9913, Software and Cyberinfrastructure for Astronomy IV. SPIE, Bellingham, p. 99133E

Jenkins J. M., Tenenbaum P., Seader S., Burke C. J., McCauliff S. D., Smith J. C., Twicken J. D., Chandrasekaran H., 2017, Technical Report, Kepler Science Document KSCI-19081-002, Kepler Data Processing Handbook: Transiting Planet Search NASA/Ames Research Center, Moffett Field, California

Jensen E., 2013, Astrophysics Source Code Library, record ascl:1306.007

Johnson D. R. H., Soderblom D. R., 1987, AJ, 93, 864

Johnson J. A. et al., 2012, AJ, 143, 111

Kempton E. M. R. et al., 2018, PASP, 130, 114401

Kennedy G. M., Kenyon S. J., 2008, ApJ, 673, 502

Kipping D. M., 2013, MNRAS, 435, 2152

Klein B. et al., 2021, MNRAS, 502, 188

Kotani T. et al., 2018, in Evans C. J., Simard L., Takami H., eds, SPIE Conf. Ser. Vol. 10702, Ground-based and Airborne Instrumentation for Astronomy VII. SPIE, Bellingham, p. 1070211

Kovács G., Zucker S., Mazeh T., 2002, A\&A, 391, 369

Kreidberg L., 2015, PASP, 127, 1161

Kreidberg L. et al., 2019, Nature, 573, 87
Laughlin G., Bodenheimer P., Adams F. C., 2004, ApJ, 612, L73

Lester K. V. et al., 2021, AJ, 162, 75

Li J., Tenenbaum P., Twicken J. D., Burke C. J., Jenkins J. M., Quintana E. V., Rowe J. F., Seader S. E., 2019, PASP, 131, 024506

Lightkurve Collaboration et al., 2018, Astrophysics Source Code Library, record ascl: 1812.013

Liu B., Ji J., 2020, Res. Astron. Astrophys., 20, 164

Liu B., Lambrechts M., Johansen A., Liu F., 2019, A\&A, 632, A7

Ma B., Ge J., 2014, MNRAS, 439, 2781

McLaughlin D. B., 1924, ApJ, 60, 22

Magnier E. A. et al., 2013, ApJS, 205, 20

Maldonado J. et al., 2020, A\&A, 644, A68

Mann A. W., Brewer J. M., Gaidos E., Lépine S., Hilton E. J., 2013, AJ, 145, 52

Mann A. W., Feiden G. A., Gaidos E., Boyajian T., von Braun K., 2015, ApJ, 804,64

Mann A. W. et al., 2019, ApJ, 871, 63

Mao S., Paczynski B., 1991, ApJ, 374, L37

Marcy G. W., Butler R. P., Fischer D., Vogt S. S., Lissauer J. J., Rivera E. J., 2001, ApJ, 556, 296

Marsh T. R., 2001, in Boffin H. M. J., Steeghs D., Cuypers J., eds, Lecture Notes in Physics, Vol. 573, Astrotomography, Indirect Imaging Methods in Observational Astronomy. Springer-Verlag, Berlin, p. 1

Masci F. J. et al., 2019, PASP, 131, 018003

Matson R. A., Howell S. B., Horch E. P., Everett M. E., 2018, AJ, 156, 31

Morales J. C. et al., 2019, Science, 365, 1441

Moutou C. et al., 2017, MNRAS, 472, 4563

Moutou C. et al., 2020, A\&A, 642, A72

Narita N. et al., 2015, J. Astron. Telesc. Instrum. Syst., 1, 045001

Narita N. et al., 2019, J. Astron. Telesc. Instrum. Syst., 5, 015001

Newton E. R., Charbonneau D., Irwin J., Berta-Thompson Z. K., Rojas-Ayala B., Covey K., Lloyd J. P., 2014, AJ, 147, 20

Newton E. R., Mondrik N., Irwin J., Winters J. G., Charbonneau D., 2018, AJ, 156, 217

Parviainen H., Aigrain S., 2015, MNRAS, 453, 3821

Parviainen H. et al., 2020, A\&A, 633, A28

Parviainen H. et al., 2021, A\&A, 645, A16

Pecaut M. J., Mamajek E. E., 2013, ApJS, 208, 9

Pepper J. et al., 2007, PASP, 119, 923

Pepper J., Kuhn R. B., Siverd R., James D., Stassun K., 2012, PASP, 124, 230

Petigura E. A. et al., 2018, AJ, 155, 89

Pollacco D. L. et al., 2006, PASP, 118, 1407

Pollack J. B., Hubickyj O., Bodenheimer P., Lissauer J. J., Podolak M., Greenzweig Y., 1996, Icarus, 124, 62

Rayner J. T., Toomey D. W., Onaka P. M., Denault A. J., Stahlberger W. E., Vacca W. D., Cushing M. C., Wang S., 2003, PASP, 115, 362

Rayner J. T., Onaka P. M., Cushing M. C., Vacca W. D., 2004, in Moorwood A. F. M., Iye M., eds, SPIE Conf. Ser. Vol. 5492, Ground-based Instrumentation for Astronomy. SPIE, Bellingham, p. 1498

Rayner J. T., Cushing M. C., Vacca W. D., 2009, ApJS, 185, 289

Ricker G. R. et al., 2014, in Oschmann J., Jacobus M., Clampin M., Fazio G. G., MacEwen H. A., eds, SPIE Conf. Ser. Vol. 9143, Space Telescopes and Instrumentation 2014: Optical, Infrared, and Millimeter Wave. SPIE, Bellingham, p. 914320

Ricker G. R. et al., 2015, J. Astron. Telesc. Instrum. Syst., 1, 014003

Rodriguez J. E. et al., 2020, AJ, 160, 117

Rossiter R. A., 1924, ApJ, 60, 15

Santos N. C., Israelian G., Mayor M., 2004, A\&A, 415, 1153

Schlegel D. J., Finkbeiner D. P., Davis M., 1998, ApJ, 500, 525

Schlieder J. E. et al., 2021, Frontiers Astron. Space Sci., 8, 63

Seifahrt A., Stürmer J., Bean J. L., Schwab C., 2018, in Evans C. J., Simard L., Takami H., eds, SPIE Conf. Ser. Vol. 10702, Ground-based and Airborne Instrumentation for Astronomy VII. SPIE, Bellingham, p. 107026D

Skrutskie M. F. et al., 2006, AJ, 131, 1163

Smith J. C. et al., 2012, PASP, 124, 1000 
Sousa S. G., Santos N. C., Israelian G., Mayor M., Udry S., 2011, A\&A, 533, A141

Speagle J. S., 2020, MNRAS, 493, 3132

Stassun K. G., Torres G., 2016, AJ, 152, 180

Stassun K. G., Torres G., 2021, ApJ, 907, L33

Stassun K. G., Collins K. A., Gaudi B. S., 2017, AJ, 153, 136

Stassun K. G., Corsaro E., Pepper J. A., Gaudi B. S., 2018a, AJ, 155, 22

Stassun K. G. et al., 2018b, AJ, 156, 102

Stassun K. G. et al., 2019, AJ, 158, 138

Stumpe M. C. et al., 2012, PASP, 124, 985

Stumpe M. C., Smith J. C., Catanzarite J. H., Van Cleve J. E., Jenkins J. M.,

Twicken J. D., Girouard F. R., 2014, PASP, 126, 100

Torres G., Andersen J., Giménez A., 2010, A\&AR, 18, 67

Trotta R., 2008, Contemp. Phys., 49, 71

Twicken J. D. et al., 2018, PASP, 130, 064502

Vanderspek R. et al., 2019, ApJ, 871, L24
Wheatley P. J. et al., 2018, MNRAS, 475, 4476

Wright E. L. et al., 2010, AJ, 140, 1868

Xu X., Cisewski-Kehe J., Davis A. B., Fischer D. A., Brewer J. M., 2019, AJ, 157,243

Zang W. et al., 2018, AJ, 156, 236

Zang W. et al., 2020, ApJ, 897, 180

Zang W. et al., 2021, Res. Astron. Astrophys., 21, 239

Zechmeister M., Kürster M., 2009, A\&A, 496, 577

Zechmeister M. et al., 2018, A\&A, 609, A12

Zhou G. et al., 2019, AJ, 158, 141

\section{APPENDIX A: PRIOR SETTINGS FOR TESS-ONLY FIT, GROUND PHOTOMETRIC DATA DETRENDING AND RV-ONLY MODELLING}

Table A1. Prior settings and posterior values for the fit to the TESS only data.

\begin{tabular}{lccc}
\hline Parameter & Best-fitting Value & Prior & Description \\
\hline Planetary parameters & $6.38758_{-0.00003}^{+0.00003}$ & $\mathcal{U}(6.2,6.6)$ & Orbital period of TOI-530b \\
$P_{\mathrm{b}}(\mathrm{d})$ & $1470.201_{-0.003}^{+0.002}$ & $\mathcal{U}(1468,1472)$ & Mid-transit time of TOI-530b \\
$T_{0, \mathrm{~b}}(\mathrm{BJD}-2457000)$ & $0.436_{-0.070}^{+0.078}$ & $\mathcal{U}(0,1)$ & Parametrization for $p$ and $b$ \\
$r_{1, \mathrm{~b}}$ & $0.152_{-0.004}^{+0.004}$ & $\mathcal{U}(0,1)$ & Parametrization for $p$ and $b$ \\
$r_{2, \mathrm{~b}}$ & 0 & Fixed & Orbital eccentricity of TOI-530b \\
$e_{\mathrm{b}}$ & 90 & Fixed & Argument of periapsis of TOI-530b \\
$\omega_{\mathrm{b}}\left({ }^{\mathrm{o}}\right)$ & $4702_{-206}^{+207}$ & $\mathcal{J}\left(100,100^{2}\right)$ & Stellar density \\
Stellar parameters & 1 & Fixed & TESS photometric dilution factor \\
$\rho_{*}\left(\mathrm{~kg} \mathrm{~m}{ }^{-3}\right)$ & $-0.000009_{-0.00009}^{+0.00009}$ & $\mathcal{N}\left(0,0.1^{2}\right)$ & Mean out-of-transit flux of TESS photometry \\
$T E S S$ photometry parameters & $0.03_{-0.02}^{+15.11}$ & $\mathcal{J}\left(10^{-6}, 10^{6}\right)$ & TESS additive photometric jitter term \\
$D_{\mathrm{TESS}}$ & $0.31_{-0.17}^{+0.28}$ & $\mathcal{U}(0,1)$ & Quadratic limb-darkening coefficient \\
$M_{\text {TESS }}$ & $0.42_{-0.25}^{+0.32}$ & $\mathcal{U}(0,1)$ & Quadratic limb-darkening coefficient \\
$\sigma_{\mathrm{TESS}}(\mathrm{ppm})$ & $q_{1}$ & \\
$q_{2}$ & &
\end{tabular}

Table A2. Prior settings for detrending the ground data.

\begin{tabular}{lcl}
\hline Parameter & Prior & \multicolumn{1}{c}{ Description } \\
\hline Planetary parameters & & \\
$P_{b}(\mathrm{~d})$ & $\mathcal{U}(6.386,6.388)$ & Orbital period of TOI-530b \\
$T_{0, b}$ (BJD-2457000) & $\mathcal{U}(1470.198,1470.204)$ & Mid-transit time of TOI-530b \\
$r_{1, b}$ & $\mathcal{U}(0.4,0.7)$ & Parametrization for $p$ and $b$ \\
$r_{2, b}$ & $\mathcal{U}(0.13,0.17)$ & Parametrization for $p$ and $b$ \\
$e_{b}$ & 0 (Fixed) & Orbital eccentricity of TOI-530b \\
$\omega_{b}(\mathrm{deg})$ & 90 (Fixed) & Argument of periapsis of TOI-530b \\
Stellar parameters & $\mathcal{N}\left(4702,207^{2}\right)$ & Stellar density \\
$\rho_{*}\left(\mathrm{~kg} \mathrm{~m} \mathrm{~m}^{-3}\right)$ & & \\
Photometry parameters for each ground light & & \\
curve & $1($ Fixed $)$ & Photometric dilution factor \\
$D_{i}$ & $\mathcal{N}\left(0,0.1^{2}\right)$ & Mean out-of-transit flux of ground photometry \\
$M_{i}$ & $\mathcal{J}\left(10^{-1}, 10^{5}\right)$ & Ground additive photometric jitter term \\
$\sigma_{i}(\mathrm{ppm})$ & $\mathcal{U}(0,1)$ & Linear limb-darkening coefficient \\
$q_{i}$ & & \\
\hline
\end{tabular}


Table A3. Prior settings and posteriors of RV-only modelling.

\begin{tabular}{lccc}
\hline Parameter & Priors & Best fitting & Description \\
\hline Planetary parameters & & & Orbital period of TOI-530b \\
$P_{\mathrm{b}}(\mathrm{d})$ & 6.38758 (Fixed) & 6.38758 & Mid-transit time of TOI-530b \\
$T_{0, \mathrm{~b}}(\mathrm{BJD})$ & 1470.201 (Fixed) & 1470.201 & Orbital eccentricity of TOI-530b \\
$e$ & 0 (Fixed) & 0 & Argument of periapsis of TOI-530b \\
$\omega$ & 90 (Fixed) & 90 & Linear RV slope for SPIRou \\
$R V$ parameters & $\mathcal{U}(0,100)$ & $21.2_{-2.5}^{+2.5}$ & Systemic velocity for SPIRou \\
$\dot{\gamma}\left(\mathrm{m} \mathrm{s}^{-1} \mathrm{~d}^{-1}\right)$ & $\mathcal{U}(-150,150)$ & $-28.1_{-9.6}^{+9.6}$ & Extra jitter term for SPIRou \\
$\mu_{\text {SPIRou }}\left(\mathrm{m} \mathrm{s}^{-1}\right)$ & $\mathcal{J}(0.1,100)$ & $30.5_{-6.7}^{+8.8}$ & RV semi-amplitude of TOI-530b \\
$\sigma_{\text {SPIRou }}\left(\mathrm{m} \mathrm{s}^{-1}\right)$ & $\mathcal{U}(0,200)$ & $61.3_{-12.4}^{+12.2}$ & \\
$K_{\mathrm{b}}\left(\mathrm{m} \mathrm{s}^{-1}\right)$ & & &
\end{tabular}

${ }^{1}$ Department of Astronomy, Tsinghua University, Beijing 100084, People's Republic of China

${ }^{2}$ Department of Physics, Tsinghua University, Beijing 100084, People's Republic of China

${ }^{3}$ National Astronomical Observatories, Chinese Academy of Sciences, $20 \mathrm{~A}$ Datun Road, Chaoyang District, Beijing 100012, People's Republic of China

${ }^{4}$ Canada-France-Hawaii Telescope, CNRS, Kamuela, HI 96743, USA

${ }^{5}$ Univ. de Toulouse, CNRS, IRAP, 14 Avenue Belin, F-31400 Toulouse, France

${ }^{6}$ School of Aerospace Engineering, Tsinghua University, Beijing 100084,

People's Republic of China

${ }^{7}$ Center for Computational Astrophysics, Flatiron Institute, 162 Fifth Ave, New York, NY 10010, USA

${ }^{8}$ Department of Physics and Astronomy, Vanderbilt University, 6301 Stevenson Center Ln., Nashville, TN 37235, USA

${ }^{9}$ Department of Physics, Fisk University, 1000 17th Avenue North, Nashville, TN 37208, USA

${ }^{10}$ Department of Astronomy, University of California Berkeley, Berkeley, CA 94720, USA

${ }^{11}$ Komaba Institute for Science, The University of Tokyo, 3-8-1 Komaba, Meguro, Tokyo 153-8902, Japan

${ }^{12}$ Instituto de Astrofísica de Canarias (IAC), Vía Láctea s/n, E-38205 La Laguna, Tenerife, Spain

${ }^{13}$ Dept. Astrofśica, Universidad de La Laguna (ULL), E-38206 La Laguna, Tenerife, Spain

${ }^{14}$ NASA Exoplanet Science Institute, Caltech/IPAC, Mail Code 100-22, 1200

E. California Blvd., Pasadena, CA 91125, USA

${ }^{15}$ NASA Ames Research Center, Moffett Field, CA 94035, USA

${ }^{16}$ Center for Astrophysics | Harvard \& Smithsonian, 60 Garden Street, Cambridge, MA 02138, USA

${ }^{17}$ Department of Physics and Kavli Institute for Astrophysics and Space Research, Massachusetts Institute of Technology, Cambridge, MA 02139, USA

${ }^{18}$ NASA Goddard Space Flight Center, 8800 Greenbelt Road, Greenbelt, MD 20771, USA
${ }^{19}$ University of Maryland, Baltimore County, 1000 Hilltop Circle, Baltimore, MD 21250, USA

${ }^{20}$ Department of Physics \& Astronomy, University of Kansas, $1082 \mathrm{Mal}$ ott,1251 Wescoe Hall Dr., Lawrence, KS 66045, USA

${ }^{21}$ Department of Physics and Astronomy, Embry-Riddle Aeronautical University, 3700 Willow Creek Rd, Prescott, AZ 86301, USA

${ }^{22}$ El Sauce Observatory, Coquimbo, 1870000, Chile

${ }^{23}$ Department of Astronomy and Astrophysics, University of California, Santa Cruz, CA 95064, USA

${ }^{24}$ Department of Astronomy, California Institute of Technology, Pasadena, CA 91125, USA

${ }^{25}$ Okayama Observatory, Kyoto University, 3037-5 Honjo, Kamogatacho, Asakuchi, Okayama 719-0232, Japan

${ }^{26}$ Department of Multi-Disciplinary Sciences, Graduate School of Arts and Sciences, The University of Tokyo, 3-8-1 Komaba, Meguro, Tokyo 153-8902, Japan

${ }^{27}$ Department of Earth and Planetary Science, Graduate School of Science, The University of Tokyo, 7-3-1 Hongo, Bunkyo-ku, Tokyo 113-0033, Japan

${ }^{28}$ Zhejiang Institute of Modern Physics, Department of Physics \& Zhejiang University-Purple Mountain Observatory Joint Research Center for Astronomy, Zhejiang University, Hangzhou 310027, People's Republic of China

${ }^{29}$ Department of Astronomy, The University of Tokyo, 7-3-1 Hongo, Bunkyoku, Tokyo 113-0033, Japan

${ }^{30}$ U.S. Naval Observatory, Washington, DC 20392, USA

${ }^{31}$ Japan Science and Technology Agency, PRESTO, 3-8-1 Komaba, Meguro, Tokyo 153-8902, Japan

${ }^{32}$ Astrobiology Center, 2-21-1 Osawa, Mitaka, Tokyo 181-8588, Japan

${ }^{33}$ Department of Earth, Atmospheric and Planetary Science, Massachusetts Institute of Technology, 77 Massachusetts Avenue, Cambridge, MA 02139, USA

${ }^{34}$ Space Telescope Science Institute, 3700 San Martin Drive, Baltimore, MD 21218, USA

${ }^{35}$ Department of Aeronautics and Astronautics, MIT, 77 Massachusetts Avenue, Cambridge, MA 02139, USA

${ }^{36}$ Department of Astrophysical Sciences, Princeton University, 4 Ivy Lane, Princeton, NJ 08544, USA

This paper has been typeset from a $\mathrm{T}_{\mathrm{E}} \mathrm{X} / \mathrm{L} \mathrm{A} \mathrm{E} \mathrm{X}$ file prepared by the author. 